\title{
Les « cahiers manuscrits » d'Etienne Sigaut : jonques et sampans chinois
}

The Notebooks (1943-1947) of Etienne Sigaut: chinese junks and sampans

Los cuardernos manuscritos de Etienne Sigaut : juncos y sampanes chinos

\section{Eric Rieth}

\section{OpenEdition}

\section{Journals}

Édition électronique

URL : https://journals.openedition.org/tc/285

DOI : $10.4000 /$ tc. 285

ISSN : 1952-420X

\section{Éditeur}

Éditions de l'EHESS

\section{Édition imprimée}

Date de publication : 1 janvier 2001

Pagination : 141-174

ISSN : 0248-6016

\section{Référence électronique}

Eric Rieth, «Les « cahiers manuscrits » d'Etienne Sigaut : jonques et sampans chinois », Techniques \& Culture [En ligne], 35-36 | 2001, mis en ligne le 07 septembre 2012, consulté le 29 septembre 2022.

URL : http://journals.openedition.org/tc/285; DOI : https://doi.org/10.4000/tc.285

Ce document a été généré automatiquement le 29 septembre 2022.

Tous droits réservés 


\title{
Les « cahiers manuscrits » d'Etienne Sigaut : jonques et sampans chinois
}

\author{
The Notebooks (1943-1947) of Etienne Sigaut: chinese junks and sampans \\ Los cuardernos manuscritos de Etienne Sigaut : juncos y sampanes chinos
}

\section{Eric Rieth}

Je remercie Jérôme Legrand, documentaliste au service recherche du Musée national de la Marine, chargé des collections photographiques, de son aide efficace et de sa connaissance du fonds Sigaut. Je remercie également Annie Madet, conservateur-adjoint au service des collections du Musée national de la Marine, chargée du fonds iconographique où sont conservés les « cahiers manuscrits » de Sigaut. Sa collaboration a été précieuse.

1 Est présentée ici une source inédite conservée au Musée national de la Marine de Paris. Il s'agit des «cahiers manuscrits » d'Etienne Sigaut (1887-1983)², qui constituent un ensemble documentaire exceptionnel pour la connaissance de l'architecture navale traditionnelle chinoise ${ }^{3}$. Avant d'examiner en détail cette documentation, il importe, dans une première partie, de situer brièvement l'auteur de ces « cahiers manuscrits ».

2 E. Sigaut est né à Saint-Servan (Ille-et-Vilaine) le 3 décembre 1887. Son père, lieutenant au 15ème bataillon d'artillerie de forteresse, était alors en garnison dans cette petite cité bretonne proche de Saint-Malo. Licencié en droit, diplômé de l'École supérieure de commerce de Dijon, Sigaut commença sa carrière à la Compagnie des Messageries Maritimes $^{4}$ le 15 octobre 1908 en tant que surnuméraire chez M.M. Gelattly, Hankey et Cie, correspondants à Anvers des Messageries. Comme commis de 3ème classe par intérim, il effectua un premier séjour à Shanghaï du 30 mars 1911 au 14 février 1912. Son second séjour débuta en 1923. Le 11 décembre de cette année-là, E. Sigaut fut nommé agent de 3ème classe faisant fonction d'agent général. Le 1er janvier 1926, il était promu agent général de 2ème classe, "faisant fonction d'agent général pour la zone d'Extrême Orient", poste qu'il occupa jusqu'au 11 février 1928. De retour en France, il travailla à Paris du 20 septembre au 2 novembre 1928. Le 29 janvier 1929, Sigaut démissionna de la Compagnie des Messageries Maritimes, «libre de tout engagement, alors qu'il se rendait dans la zone de l'Océan Indien pour y occuper les 
fonctions d'agent général », selon les termes de l'attestation du chef du service du personnel des Messageries Maritimes.

Deux photographies ${ }^{5}$, dont l'une datée de décembre 1926, première année de fonction de Sigaut comme agent général de 2ème classe, montrent un homme dans sa pleine maturité (il est alors âgé de 39 ans), au front dégarni, à la fine moustache soigneusement taillée, à l'allure élégante et bourgeoise (fig. 1). Outre son activité professionnelle, l'agent général était un personnage important au sein de la petite communauté européenne expatriée vivant à des milliers de kilomètres de son pays natal.

4 À cette époque, la Compagnie des Messageries Maritimes, dont la ligne MarseilleShanghaï avait été inaugurée en avril 1896, comportait, outre l'agence générale de Marseille et l'agence d'armement de Dunkerque, vingt-neuf agences réparties en six zones ayant chacune à leur tête une agence générale. Celle de la zone d'Extrême-Orient était située à Shanghaï, cité portuaire de première importance localisée sur le fleuve Huang-p'u, à une trentaine de kilomètres de son confluent avec le mythique fleuve Yang-tsé.

5 Lorsque E. Sigaut démissionna de la Compagnie des Messageries Maritimes, il n'abandonna pas pour autant la Chine et Shanghaï. À une date que nous ignorons, il retourna en Chine pour y demeurer, semble-t-il, jusque dans les années $1947^{6}$. Il semblerait alors avoir été engagé dans des activités commerciales et financières. Insérées dans les "cahiers", de nombreuses quittances à l'en-tête de différentes sociétés (anglaises et françaises) localisées en Chine sont couvertes de croquis et de notes de Sigaut. On peut supposer que ces documents correspondent aux entreprises pour lesquelles il travaillait.

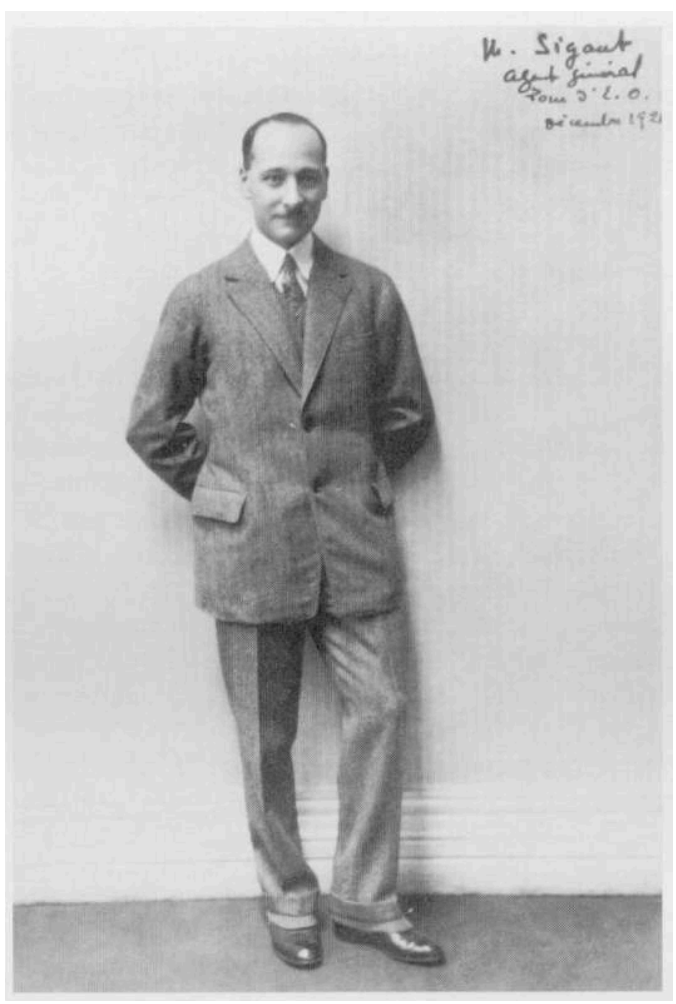

Figure 1. Portrait officiel de l'agent général des Messageries Maritimes Etienne Sigaut, décembre 1926 (cliché Musée national de la Marine) 
6 La période que Sigaut traversa, comme agent général des Messageries et comme résidant à Shanghaï, fut particulièrement mouvementée pour l'histoire de la Chine. En 1921, en effet, était fondé à Shanghaï le parti communiste chinois. En 1927, les militants communistes prirent la ville et remirent le pouvoir à Chiang Kai-Shek. Rompant son alliance avec les communistes, le leader nationaliste fit exécuter de nombreux militants. Comment ne pas rappeler, en évoquant cette phase de l'histoire de la Chine, les figures de Tchen, Katow ou Kyo qui parcourent, telles les ombres d'une tragédie, $L a$ condition humaine, publiée par Malraux en 1933 ? Un an auparavant, la ville stratégique de Shanghaï était attaquée par les Japonais qui imposèrent un régime de terreur. Il fallut attendre la capitulation du Japon le 14 août 1945 pour voir la cité portuaire retrouver, enfin, un semblant de paix après une longue période de souffrances.

7 C'est durant ces années de conflits internes et externes que Sigaut, désormais libéré de son poste d'agent général de la Compagnie des Messageries Maritimes, se lança dans l'étude de l'architecture navale chinoise. À Shanghaï, il eut l'occasion de rencontrer et de devenir l'ami- de G.R.G. Worcester, considéré, aujourd'hui encore, comme l'un des meilleurs connaisseurs de l'histoire récente des jonques et des sampans et, plus particulièrement, de ceux du Yang-tsé.

8 Worcester, ancien officier de la Royal Navy, était en poste à Shanghaï au service des douanes maritimes chinoises. En 1943, après la prise de la ville par les Japonais, il fut arrêté et passa plusieurs années dans un camp de prisonniers aux conditions de vie effroyablement sévères. Auparavant, il publia en 1940, comme assistant river inspector, un premier ouvrage portant sur les jonques et sampans du haut Yang-tsé. Après sa libération, il fut l'auteur, en tant que river inspector, d'un second volume (1947-48) consacré aux jonques et sampans de l'estuaire du Yang-tsé et de la région de Shanghaï7. Ces deux livres, dont la réalisation fut encouragée et soutenue par la direction générale des douanes maritimes chinoises, ont été ensuite complétés par d'autres publications résultant de ses nombreuses enquêtes de terrain ${ }^{8}$

9 Les ouvrages et articles de Worcester contiennent de très nombreux plans de bâtiments et d'embarcations tracés par un dessinateur chinois, un certain Chang Ter Tsing (Worcester $1940: \mathrm{pl}$. XV). Ces relevés, habilement dessinés, se présentent, en règle générale, sous la forme de vues en plan, en élévation, de face (avant et arrière) et en coupes (longitudinale et transversale) visualisant les aménagements (notamment la position des cloisons transversales). Ces documents rigoureux apportent, cependant, très peu d'informations sur la structure architecturale des coques. Ce regard secondaire sur «l'anatomie » architecturale se retrouve, en l'occurrence, dans le contenu des publications. Il est vrai que c'est avant tout en tant que sailor (Worcester 1940: pl. $\mathrm{XXVI}$ ), selon son propre qualificatif, que Worcester définissait son approche des bateaux et non comme chercheur désireux de décrire dans le détail des systèmes de construction. C'est sans doute cette manière d'appréhender l'architecture navale qui conduisit Jean Poujade à juger avec une certaine sévérité cette documentation graphique qui demeure, de notre point de vue, d'un grand intérêt en dépit, il est vrai, de certaines lacunes :

«[...] les plans sont nettement insuffisants. Ils donnent des silhouettes et des coupes bien incomplètes. Les procédés de construction n'apparaissent pas; les détails de charpente sont passés sous silence » (Poujade n.d. : 51).

Peu importe pour notre propos ce jugement de J. Poujade. L'aspect majeur que nous souhaitons ici souligner concerne les liens de travail et d'amitié qui unirent, durant 
leur séjour en Chine, et après leur retour en Europe, Worcester et Sigaut. À cet égard, les termes employés par Worcester dans ses remerciements publiés lors de la parution de son second ouvrage, en 1947, sont révélateurs de la force de cette relation :

«In perfecting the various sections, others outside the Service has graciously helped either by suggestion or editorial reading. I chiefly wish to express deep gratitude to my friend Monsieur Etienne Sigaut, the scope of whose extensive knowledge of Chinese junks is only equalled by his generosity in sharing it. In the course of frequent discussions together we have been able to solve various knotty points - both literally and figuratively-, for we spent many weeks over the problem of sheeting in particular. He has, in addition, shown unfailing kindness in innumerable other ways » (Worcester 1940 : p. XVI).

11 L'insertion dans l'ouvrage de Worcester de photographies prises par Sigaut représente un autre signe de cette proximité entre les deux chercheurs (Worcester 1940: photo frontispice, pl. 18, 19). Sans doute, cet intérêt, et cette passion, pour un même sujet les bateaux traditionnels chinois-, ont-il favorisé un rapprochement intellectuel entre deux hommes isoles dans un pays en proie à la guerre, deux hommes possédant chacun une expérience professionnelle différente de l'univers maritime, Sigaut comme ancien agent général d'une grande compagnie maritime, Worcester comme inspecteur des douanes maritimes chinoises et ancien officier de marine.

L'écho de ces relations de travail est également présent dans les " cahiers manuscrits » de Sigaut. Ainsi peut-on lire, par exemple: «Le 11 novembre 1946 avec Worcester, inspecté trois vieux Hangchow trader... Worcester a pris des notes et des croquis $»^{9}$. Le 5 février 1947, Sigaut mentionne dans un de ses «cahiers manuscrits » qu'il a visité le port de Nantoo avec Worcester et son dessinateur Chang ${ }^{10}$. Une autre illustration est fournie par cette mention, à propos d'un « cahier manuscrit » portant sur l'étude d'une petite jonque de Chine du nord : «mesures prises le 7/12/47 par M. Chang (dessinateur de Worcester) »11.

$13 \mathrm{Au}$ vu de la documentation, il semble manifeste que Sigaut et Worcester ont eu, à maintes reprises, l'occasion de travailler soit directement ensemble, soit par l'intermédiaire du dessinateur de Worcester qui fut aussi celui de Sigaut à partir de 1943, année de l'emprisonnement de Worcester par les occupants japonais. Pour Sigaut comme pour son ami Worcester, les plans représentaient des documents indispensables à la connaissance des jonques et des sampans, mais constituaient également une source nécessaire à la réalisation de maquettes. Effectivement, les « cahiers manuscrits » font état, à différents endroits, de modèles réduits. En relation avec la description d'une jonque de Foochow, Sigaut note pat exemple: «[...] croquis d'une jonque de Foochow, d'après mesures prises par mon constructeur de modèles $»^{12}$. Dans un « cahier » daté du 17 août 1945, il écrit à propos de ses observations sur les jonques d'Amoy : « Documents ayant servi à la construction de mon modèle $»^{13}$. Dans des notes en date du 7 au 22 février 1947, il indique que "[son] dessinateur [est] bon observateur et lui-même constructeur de modèles $»^{14}$.

14 Cette collection de maquettes réalisées à partir des enquêtes de Sigaut existe effectivement. Elle se trouve aujourd'hui conservée au Museo storico navale de Venise, malheureusement séparée des "cahiers manuscrits» et de l'ensemble de la documentation photographique. Dans le cadre d'une exposition autour de l'œuvre de l'amiral Paris qui devait se tenir il y a quelques années au Musée national de la Marine, la présentation de quelques maquettes de la collection Sigaut, en relation avec ses « cahiers manuscrits » et ses photographies, avait été prévue. Hélas, cette exposition ne 
vit jamais le jour. Nul doute qu'elle eût fourni aussi l'occasion de faire connaître l'œuvre de Sigaut qui, pour l'essentiel, demeure inédite.

Ses publications sont en effet très rares. Deux d'entre elles sont particulièrement révélatrices de son activité scientifique. La première est une étude en anglais datée de 1960 (Sigaut est alors âgé de 73 ans), ayant pour sujet un type de jonque du nord de la Chine. Cet article, illustré par des dessins de Sigaut et un plan non signé (œuvre probable de son dessinateur chinois), est en fait la version publiée d'un des « cahiers manuscrits $»^{15}$ (figs. 2 à 6 ).

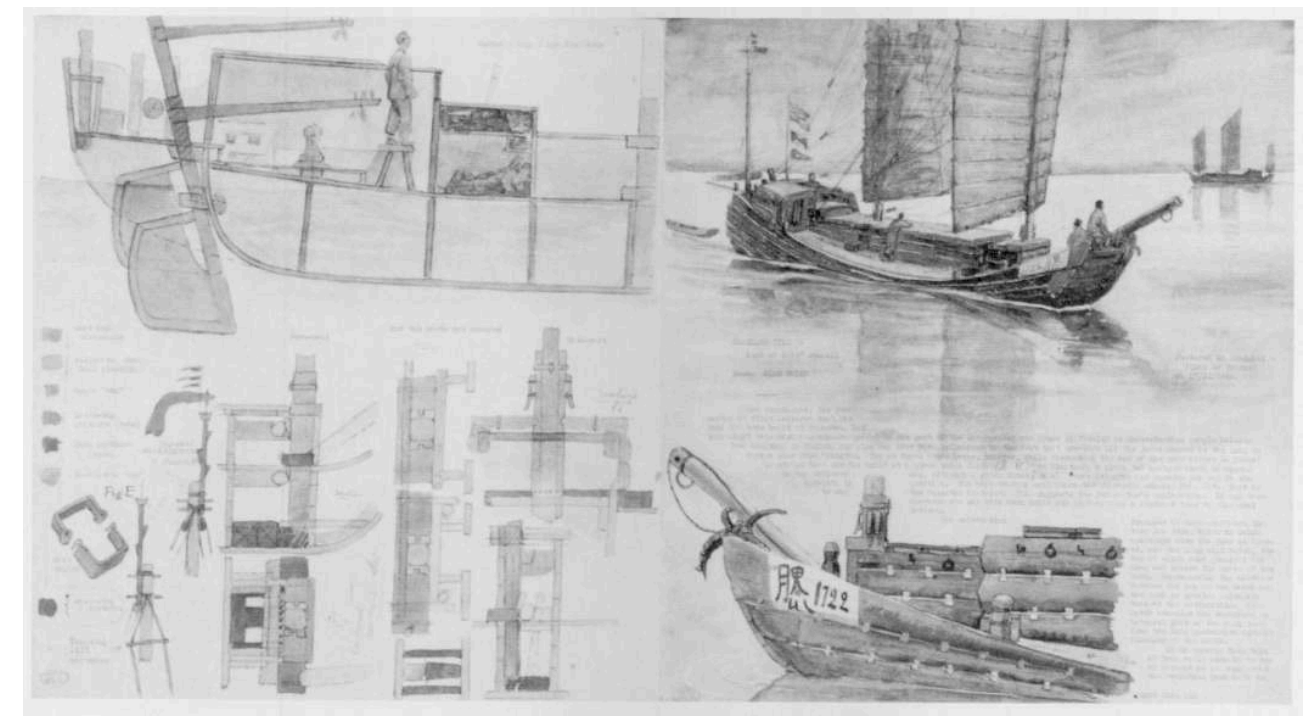

Figure 2. Jonque du nord de la Chine, " cahiers manuscrits », réf. : R9n / 42510 (1), p. 4-1, M.N.M. (cliché Musée national de la Marine)

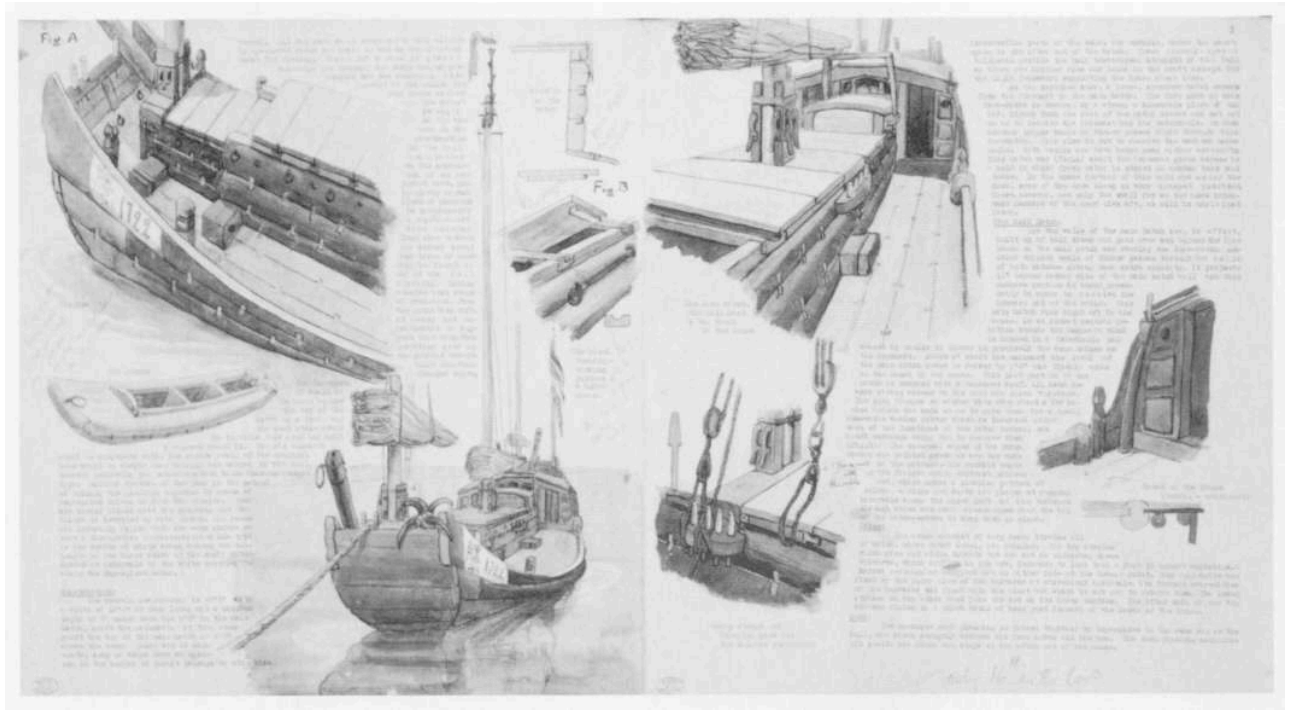

Figure 3. Jonque du nord de la Chine, « cahiers manuscrits », réf. : R9n / 42510 (1), pp. 2-3, M.N.M. (cliché Musée national de la Marine) 


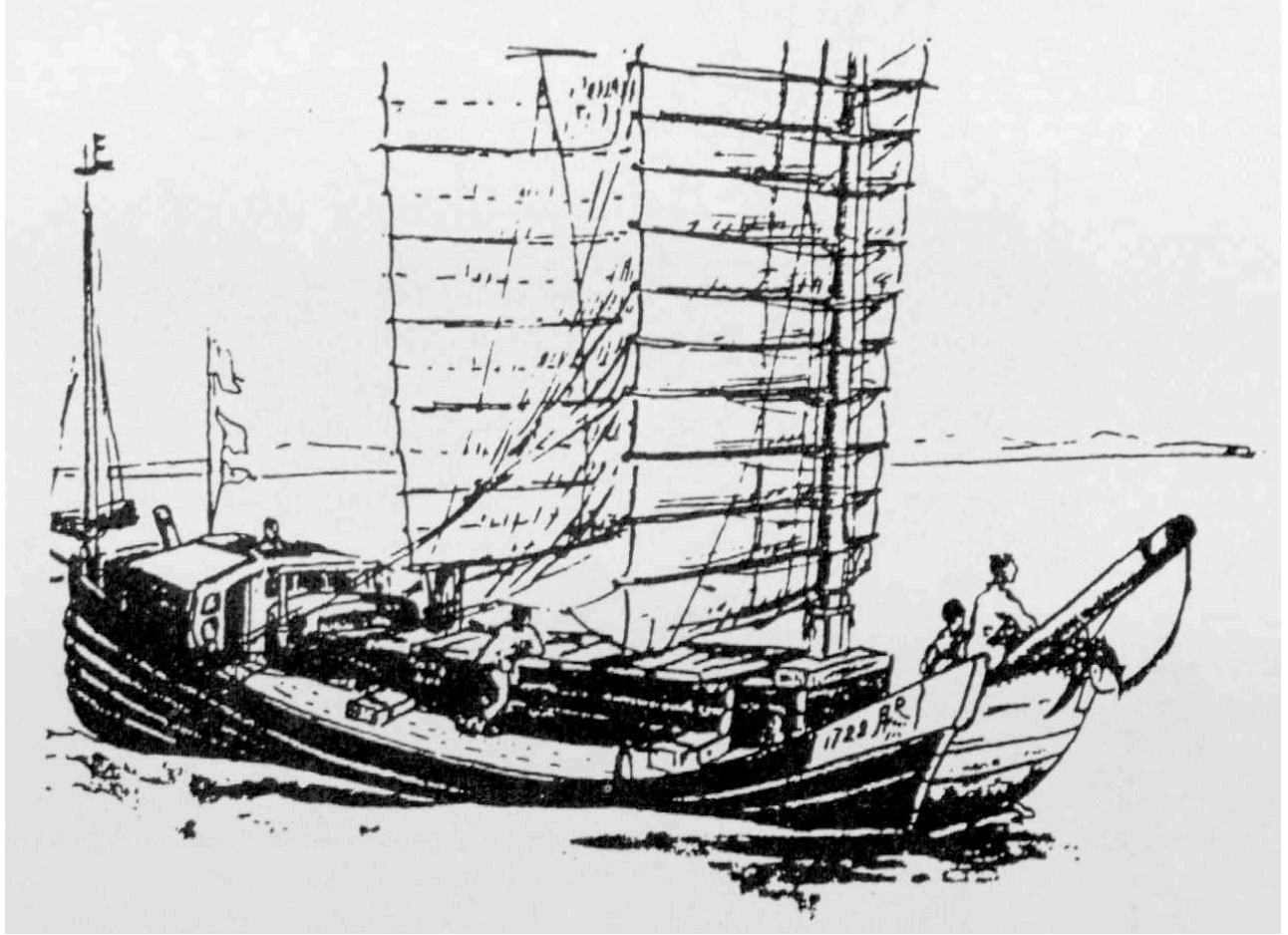

Figure 4. Jonque du nord de la Chine, vue générale ; illustration publiée dans E. Sigaut, « A northern type of Chinese junk », art. cit., p. 159

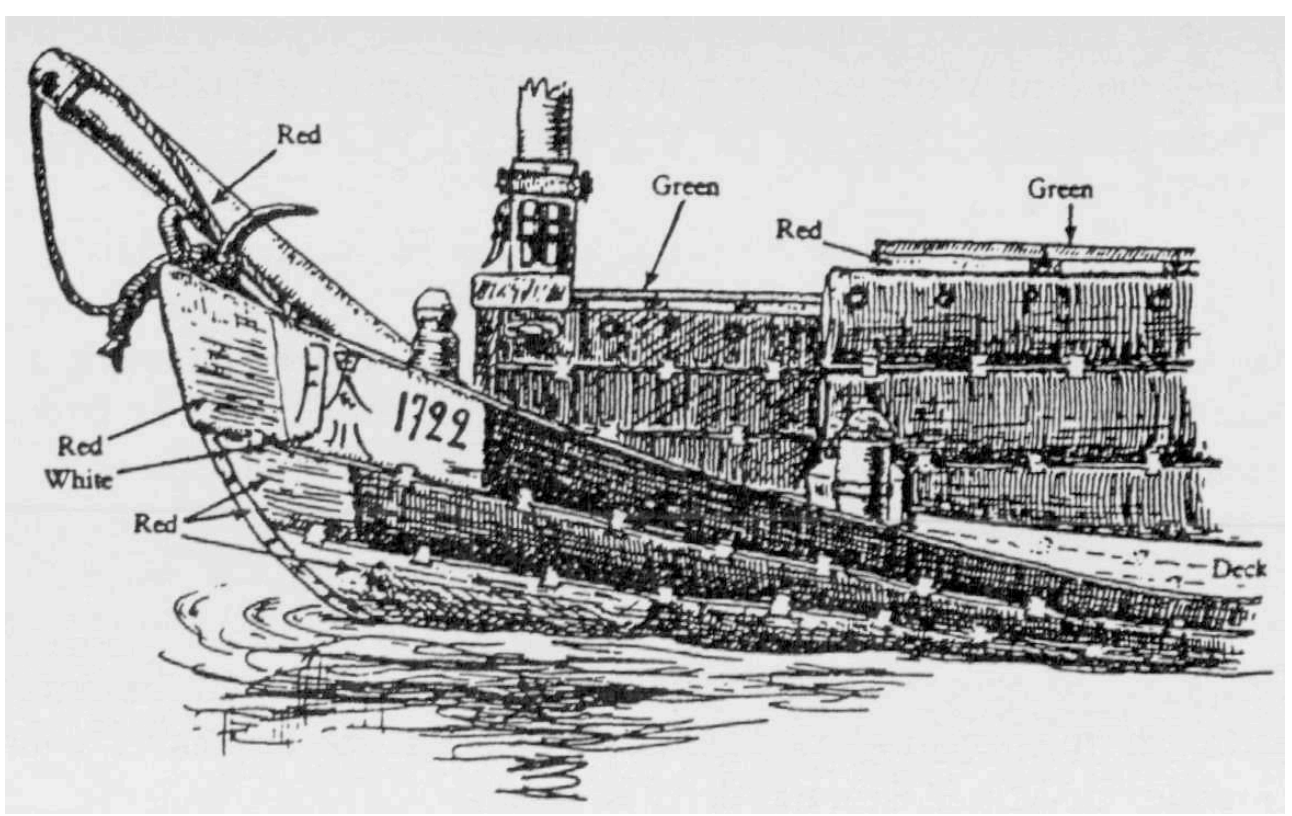

Figure 5. Jonque du nord de la Chine, vue latérale de la proue ; illustration publiée dans E. Sigaut, «A northern type of Chinese junk », art. cit., p. 162. Dans l'impossibilité de reproduire en couleurs les dessins aquerellés du « cahier manuscrit », Sigaut a indiqué par écrit les différentes couleurs. 


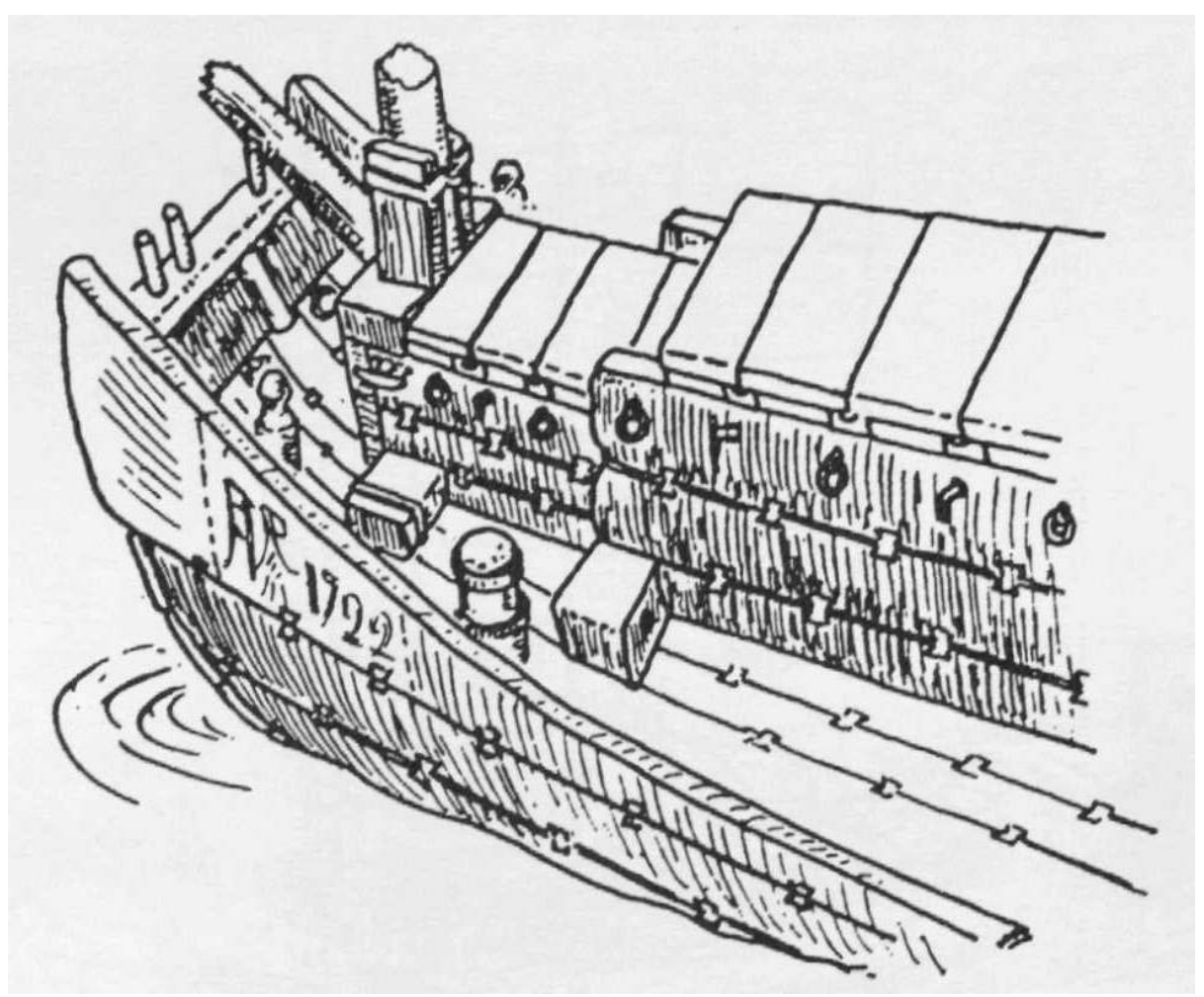

Figure 6. Jonque du nord de la Chine, vue du dessus de la proue; illustration publiée dans E. Sigaut «A northern type of Chinese junk », art. cit., p. 166

Ce « cahier » est le seul à avoir été dactylographié et rédigé en anglais. Hormis quelques phrases changées, les deux versions -manuscrite et publiée- sont similaires. Et l'on peut se demander si Sigaut n'avait pas le projet d'éditer une série d'articles dans le Mariner's Mirror, dans l'esprit de ceux publiés dans cette même revue anglaise, au rayonnement international, par son ami Worcester et par un autre grand spécialiste anglais de l'architecture navale chinoise, D. W. Waters (1938, 1939, 1940, 1946, 1955, 1961).

La seconde publication de Sigaut, également rédigée en anglais et éditée dans le Mariner's Mirror (1961), est une analyse remarquablement documentée de la vie et de l'œuvre de l'amiral Pâris ${ }^{16}$. Cette référence à Paris, le "père» de l'ethnographie nautique, ne relève probablement pas du hasard. Comme nous aurons l'occasion de le souligner ultérieurement, de nombreux points communs relient les «cahiers manuscrits » de Sigaut aux travaux ethnographiques de Pâris et, plus particulièrement, à son Essai sur la construction navale des peuples extra-européens, publié en 1843.

Tels sont, évoqués à grands traits, les quelques éléments retenus pour permettre de mieux situer E. Sigaut par rapport à son œuvre conservée, rappelons-le, au Musée national de la Marine.

Examinons à présent la nature des «cahiers manuscrits». Cette expression utilisée pour qualifier la documentation réunie par Sigaut mérite une courte explication. Ces " cahiers manuscrits » ne sont pas reliés comme de traditionnels cahiers. Il s'agit, en réalité, de grandes feuilles de papier à dessin pliées en deux au format de $30 \mathrm{~cm}$ (largeur) sur $33 \mathrm{~cm}$ (hauteur) ${ }^{17}$. Chaque feuille ainsi pliée comporte quatre pages de notes et d'illustrations. Selon l'importance des informations recueillies, chaque « cahier manuscrit », correspondant à un type (ou à une famille) de bâtiments, se compose 
d'une ou de plusieurs feuilles. En outre, un plan (parfois deux) est inséré, en règle générale, à l'intérieur de chaque "cahier». Dans le fonds iconographique du musée sont conservés cinquante-cinq "cahiers ${ }^{18}$ qui constituent autant de monographies, plus ou moins détaillées selon les cas. À l'exception d'un seul « cahier » de cinq feuilles de croquis daté de 1911 à $1929^{19}$, tous les autres ont été réalisés entre 1943 et $1947^{20}$. Rappelons qu'en 1943, Worcester avait été fait prisonnier. Selon une note publiée en 1966 dans la chronique de l'Association des Amis du Musée de la Marine, «avant d'être interné parce que sujet britannique, Mr Worcester avait fait remettre la documentation qu'il avait établie à l'intention des Douanes chinoises à Mr Sigaut. Par la suite, il l'invita à fondre leurs travaux respectifs ${ }^{21}$. La coïncidence de date entre cet épisode et le début des «cahiers manuscrits» est-elle purement fortuite ou résulte-t-elle d'une décision de Sigaut de mener à son tour, à l'exemple des recherches conduites par Worcester, des enquêtes sur les jonques et les sampans? Nul document ne permet, en vérité, de savoir ce qu'il en a été.

Les types de bâtiments étudiés par Sigaut couvrent le littoral de la Chine, entre la province du Kiang-Su au nord, et Hong-Kong au sud. Cette répartition géographique appelle deux observations principales. D'une part, les origines des bateaux de pêche et de cabotage présents dans les «cahiers » constituent un échantillonnage relativement large qui, toutefois, ne possède pas un caractère programmé. En effet, Sigaut a souvent décrit et dessiné des jonques et des sampans que les nécessités économiques ou les hasards des affrètements avaient amené à faire escale à Shanghaï. D'autre part, la majorité de la documentation se réfère à des embarcations et à des voiliers de mer. Elle vient donc compléter celle accumulée par Worcester qui portait, pour une large part, sur la batellerie du Yang-tsé.

21 Avant de revenir sur un exemple des « cahiers ", ajoutons une dernière précision. C'est en 1966 que, selon une information publiée dans la revue Neptunia, «Mr E. Sigaut, membre de l'Association [...] a fait don d'une très importante documentation sur les jonques et les autres bateaux exotiques qu'il avait rassemblée lors de ses séjours en Extrême-Orient ${ }^{22}$. Trente-quatre ans après ce don, il est apparu urgent de valoriser cette exceptionnelle mémoire écrite et visuelle d'une tradition architecturale « extraeuropéenne » aujourd'hui disparue.

L'exemple choisi est celui d'une jonque du Fu-Kien (province sud-orientale de la Chine), objet principal du « cahier manuscrit » R9n / 42 538. Ce « cahier », daté de 1945, est très représentatif, du double point de vue de sa forme et de son contenu, de l'ensemble des documents réunis par Sigaut. Il se compose de trois feuilles pliées en deux au format de $30 \mathrm{~cm}$ (largeur) sur $33 \mathrm{~cm}$ (hauteur). Deux feuilles, soit huit pages, comportent un texte écrit, avec de rares corrections, au stylo à plume, et une série d'aquarelles. Les commentaires se présentent sous la forme de notes rédigées d'une manière sommaire, sans plan bien défini. Il s'agit manifestement d'une documentation rédigée provisoirement que Sigaut avait le projet, selon ses propres termes, « de reclasser dans un ordre plus logique ${ }^{23}$. Au dos d'une feuille à l'en-tête de la Foncière et Immobilière de Chine se trouve le relevé (plan, coupe longitudinale, coupe transversale) de la jonque du Fu-Kien. Ce document au crayon est de toute évidence un brouillon. De nombreuses annotations de Sigaut corrigent ou précisent le relevé. Un second plan (coupe longitudinale, plan, section au maître-couple, élévation), mis au net sur une feuille de papier à dessin, illustre un autre modèle de jonque, originaire de la province du ChéKiang, également décrit dans le « cahier ». 
Le texte et les illustrations sont soigneusement mis en page, indice évident d'une composition, a posteriori, de notes de terrain. Effectivement, ces minutes existent et sont insérées dans le "cahier». Il s'agit principalement de croquis au crayon et de notes tracés, pour nombre d'entre eux, au dos de reçus de sociétés de commerce, International Savings Society ou China Cotton Mills, limited en particulier (fig. 7 et 8).

4 Cette manière de travailler rappelle celle du commandant Audemard (1865-1955), autre grand spécialiste de l'architecture navale chinoise, dont un album a été acquis en 1994 par le Musée national de la Marine (cf. Rieth 1995). Ce document comprenant 974 dessins, pour la plupart aquarellés, est accompagné de notes. Il est l'aboutissement très probable d'une mise au net, faite sans doute entre les années 1920 et 1940, d'observations et de croquis réalisés en Chine entre 1900 et 1913.

Reprenons la lecture, page par page, du «cahier» de Sigaut portant, en haut de la première page, la mention suivante : « Jonque du Foukien, 70 ' [pieds] au pont, hors tout env. 77', mesurée quai de France 7 mai 1945 » (fig. 9). Sigaut commence par situer les conditions de son étude avant de décrire les principales caractéristiques architecturales du bâtiment.

26 Les conditions : « Une jolie jonque du Foukien (77') est venue s'amarrer près du quai de France [situé à Shanghaii] début mai 1945 avec un chargement complet de bois de chauffage et de bambous, en grande partie en pontée [...]».

Les grandes caractéristiques architecturales : « La construction est classique. De l'avant à l'arrière la liaison longitudinale habituelle constituée par 2 serres sous de hautes hiloires, avec une ligne ininterrompue de panneaux ; coque robuste avec préceintes mais moins épaisses et moins en relief que celles du type Ningpo ou grosses jonques du Foukien. Et par dessus de très hauts pavois un peu en encorbellement, mais ici montant à peu près verticalement... alors que dans beaucoup de jonques du Foukien le frégatage est déjà fort au centre du bateau. Le tableau large en haut va en se rétrécissant jusqu'au au haut de la quille visible, le bateau lège ou légèrement échoué de l'avant [...]».

Cette description des caractéristiques architecturales externes de la jonque est complétée par trois aquarelles. Donnée importante du point de vue de la critique interne du document, Sigaut note, à propos de ces aquarelles : «Les croquis joints à cette note, pris sur place et non sur photos donnent une idée de la décoration de cette jonque, de la forme de son avant et de son arrière ». Il s'agit donc bien d'observations directes, gages d'une authenticité documentaire. Les trois pages suivantes du " cahier " décrivent et illustrent d'une façon détaillée les emménagements, certains éléments particuliers de la charpente interne et les décorations auxquelles Sigaut prête, tout au long de ses « cahiers ", une attention soutenue. 


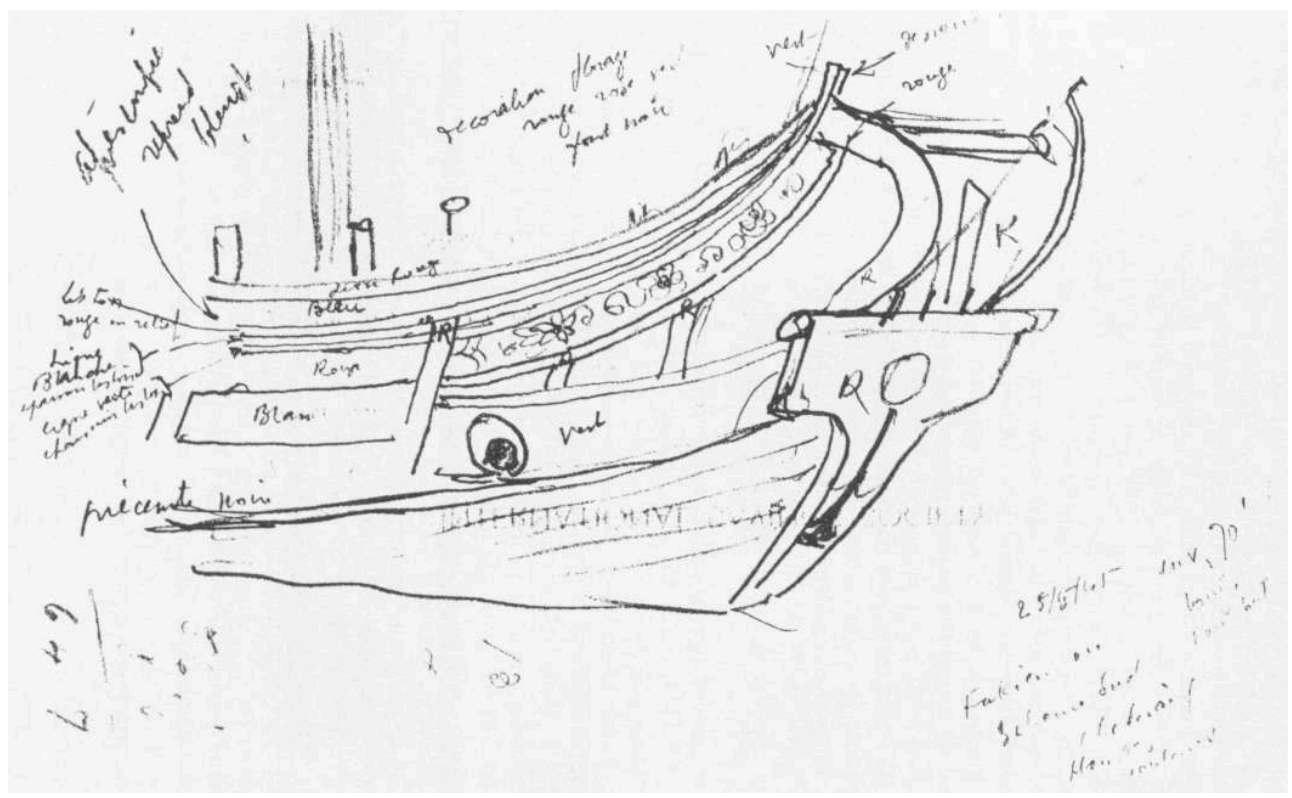

Figure 7. Croquis au crayon de la proue d'une jonque supposée être du Fu-Kien. Ce document a été réalisé au cours de l'enquête de terrain. La mise au net a été effectuée sur la feuille 2, page 1 du « cahier manuscrit » R9n / 42538

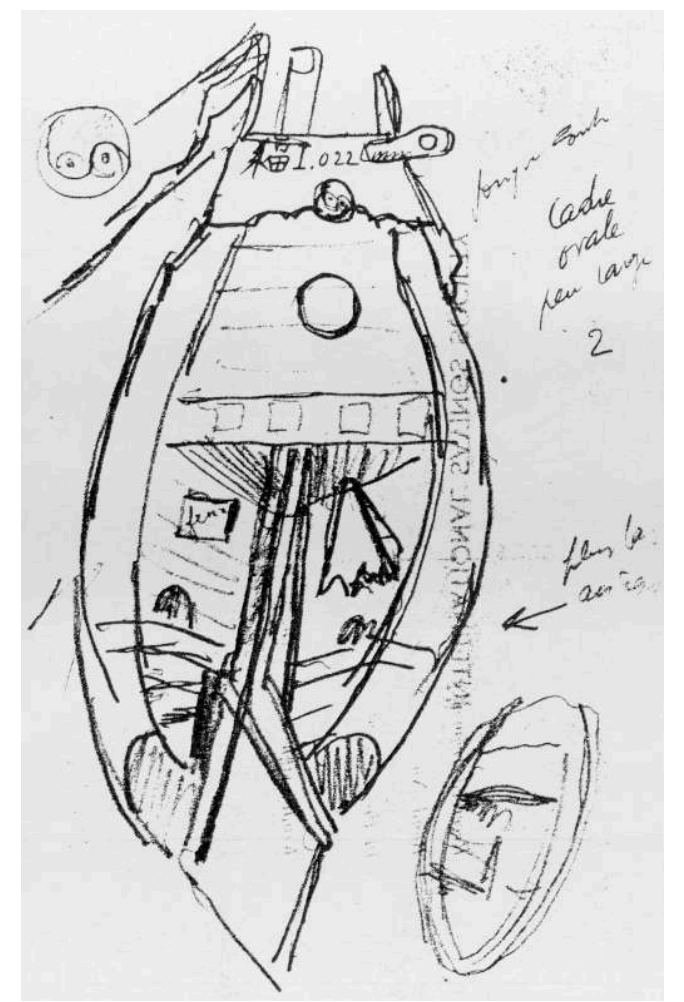

Figure 8. Croquis au crayon de la poupe d'une jonque du Fu-Kien. Pour la mise au net, $c f$. le « cahier manuscrit », R9n / 42 538, feuille 2, p. 4 


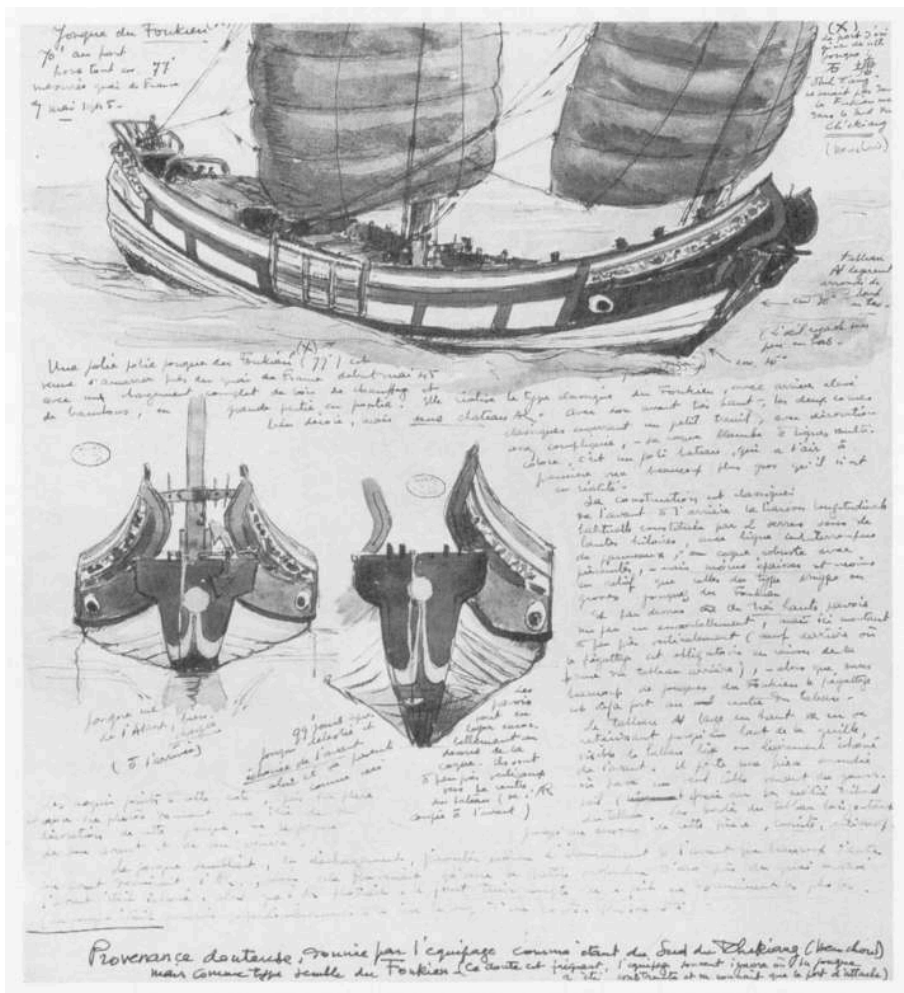

Figure 9. « cahier manuscrit » R9n / 42 538, feuille 1, page 1 (cliché Musée national de la Marine)

La double page centrale est dominée par l'élévation de la coque destinée, avant tout, à mettre en évidence les décorations peintes du bordé et du pavois (fig. 10). Ne se contentant pas seulement de reproduire les couleurs des peintures, Sigaut souligne que son aquarelle «[...] reproduit les couleurs de la jonque avec assez d'exactitude; cependant on a noté que tout n'est pas absolument identique entre tribord et bâbord [...]. Par exemple, la bande verticale orange située derrière l'œil s'arrête à bâbord au liston vert, comme sur ce dessin, mais à tribord elle montait plus haut, au moins jusqu'au liston bleu ».

30 Des autres figures composant cette double page, les plus porteuses d'informations du point de vue de la connaissance du bâtiment se réfèrent aux gaillards d'avant et d'arrière. Le gaillard d'avant est ainsi figuré à la fois en vue perspective et en coupe longitudinale. Cette dernière fait apparaître, parmi d'autres équipements, le guindeau servant à la manœuvre du câble de mouillage. De petits croquis complètent cette coupe, tels ceux représentant la manière très particulière de bosser le câble de l'ancre sur deux barres horizontales disposées sur l'avant du mât de misaine. Un commentaire vient préciser le contenu des croquis : «On se sert des barres horizontales pour bosser les câbles d'ancres ou les ancres au moyen d'erseaux. En outre ces barres qui peuvent théoriquement [souligné par E. Sigaut] pivoter sur leur axe aident au glissement des câbles allant au treuil ». Un autre croquis, tout aussi informatif, concerne l'étambrai du mât de misaine localisé au niveau du gaillard. La légende, rédigée en style télégraphique, contient des indications intéressantes: «Sur le pont du gaillard tenue du mât avant. Le tout calfaté. 2 crampons assurant la tenue des deux pièces ensembles. Renfort ordinaire à flasque au pont principal ».

31 La dernière et quatrième page de cette première feuille du "cahier » est la plus technique de toutes (fig. 11). En effet, Sigaut a dessiné et décrit avec soin la structure 
interne du pont au niveau, notamment, du gaillard d'avant et de la cale centrale de la jonque. Dans le commentaire apparaît ainsi la mention des cloisons transversales, si caractéristiques de l'architecture navale chinoise :

«Entre le grand mât et la cloison située sous le roof (non pas sous l'avant du roof, mais en arrière à lui), il y a 5 cloisons dont les 4 premières sont très rapprochées à env. 1 mètre. Ensuite des cloisons plus éloignées (env. $2 \mathrm{~m}$ ) avec boîtes à eau au dessus. Il y a alors une membrure intermédiaire. Ailleurs, quand les cloisons sont à env. $1 \mathrm{~m}$, il n'y a pas de membrures ».

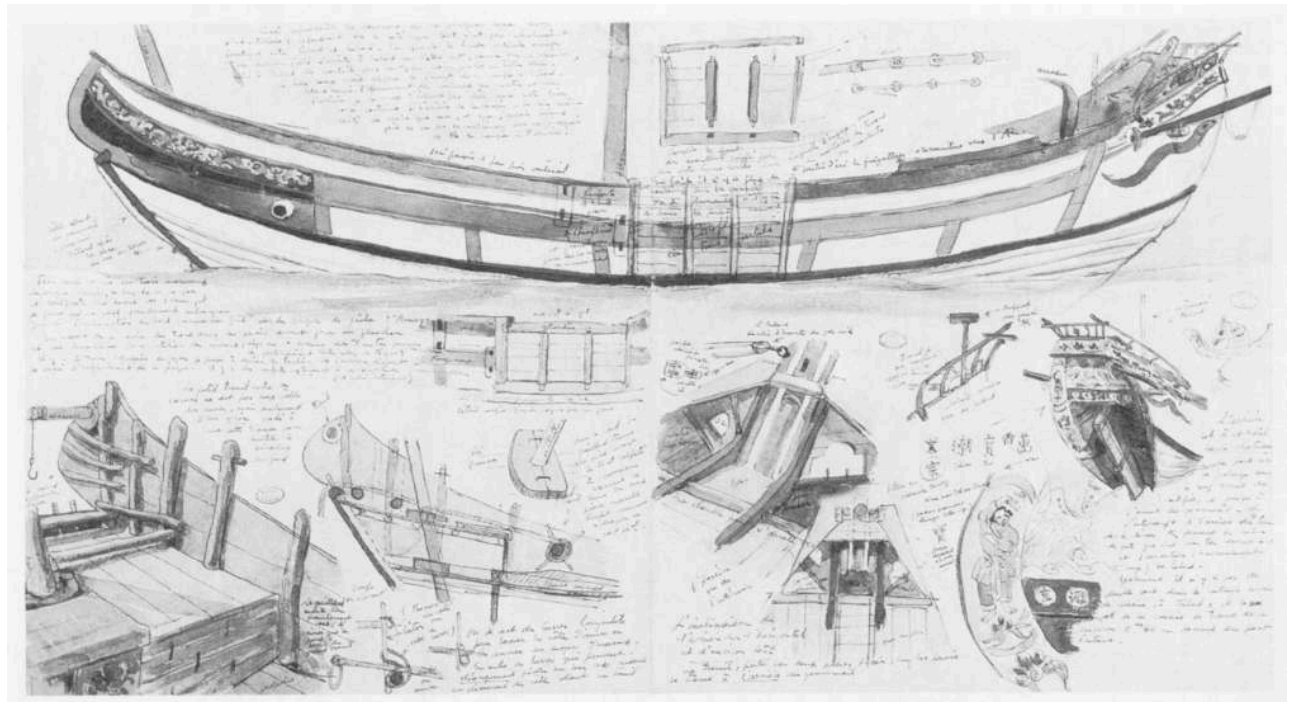

Figure 10. «cahier manuscrit » R9n / 42 538, feuille 1, double page 2-3 (cliché Musée national de la Marine)

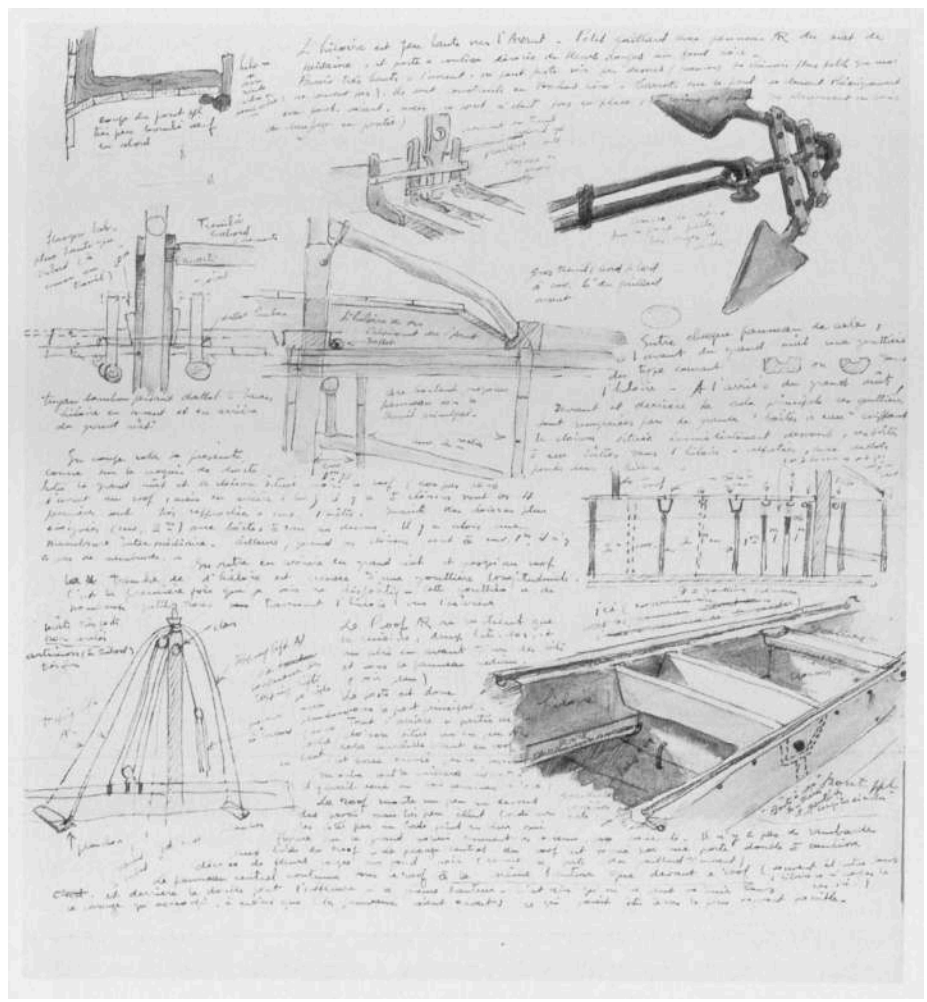

Figure 11. «cahier manuscrit » R9n / 42 538, feuille 1, double page 4 (cliché Musée national de la Marine) 
Quittant le domaine de la structure de la coque pour celui de l'organisation fonctionnelle de l'espace intérieur de la jonque, Sigaut indique que «le roof AR ne contient que la cuisine, deux lits clos et un abri en avant d'un des lits et sous le panneau médian... Le poste est donc sous le pont principal. Tout l'arrière à partir de la cloison située un peu en AR de la muraille avant du roof est donc occupé par le personnel ». Et il ajoute à ses remarques qu'il «y avait deux ou trois femmes à bord ».

$\mathrm{Au}$ regard de ces quatre pages largement illustrées figure le relevé coté (en pieds et pouces anglais) de la jonque, qui permet d'inscrire dans l'ensemble de l'architecture de la coque les différentes parties ayant donné lieu à des dessins et des commentaires de détail (fig. 12). Rappelons que ce plan est accompagné de nombreuses annotations qui montrent la rigueur avec laquelle Sigaut contrôlait et critiquait sa documentation. Un exemple révélateur de cette démarche est celui de la position de la ligne d'eau qui, sur la coupe longitudinale, apparaît parallèle à l'axe de la quille. Sigaut souligne que « quand il (son dessinateur) a mesuré, la jonque n'était pas dans ses lignes d'eau normales (mais) échouée de l'avant. À l'œil, l'avant et l'arrière dans leur partie supérieure sont à la même hauteur. En fait le pont montait un peu vers l'arrière ». La coque était donc en différence. répartissent en trois ensembles. Le premier (une page) présente un type de bateaux similaires à celui de la jonque du Fu-Kien mesurée et observée à Shanghaï le 7 mai 1945 mais dont les dimensions, le gréement ou le décor sont différents (fig. 13). Cette documentation, réunie en mai et juin 1945 à Shanghaï, accorde une place privilégiée aux décorations peintes. Pour autant, les aspects architecturaux (morphologie et structure de proue et de poupe en particulier) ne sont pas ignorés.

Le deuxième ensemble (deux pages centrales) de dessins et de commentaires se rapporte, pour une part, à une petite jonque du sud Ché-Kiang représentée en faisant route à la voile sous différentes allures (fig. 14). Aux croquis aquarellés d'ensemble s'ajoutent des dessins de détail portant sur la coupe des voiles lattées, le gréement de tête de mât, ou encore l'emplanture des mâts. Pour une autre part, ce deuxième ensemble est consacré à une "jonque Sud Ch'ckiang croquée 30 mai 1945 quai de France ", dont Sigaut s'attache à souligner le système de fixation du gouvernail axial suspendu et la forme élancée de la proue à tableau (fig. 15). Le plan, mis au net, associé à cette partie du «cahier» est également celui d'une jonque de pêche du "Sud Ch'ckiang " relevé le 10 décembre 1945 à Shanghaï (fig. 16). À cet égard, Sigaut ne justifie pas d'une manière claire et convaincante la présence, au sein d'un même « cahier », de ces types de jonques d'origine géographique différente : provinces du FuKien et du Ché-Kiang. 


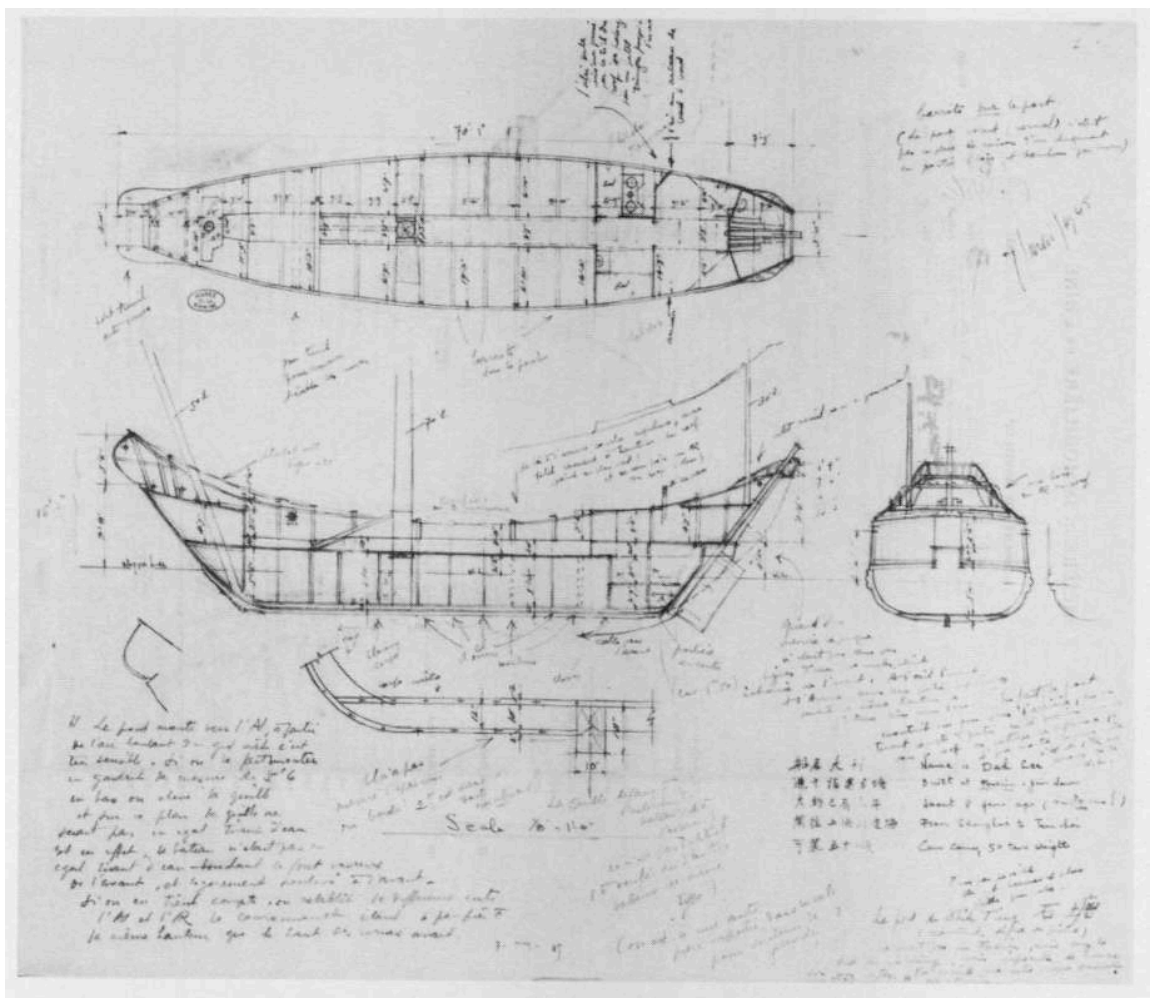

Figure 12. «cahier manuscrit » R9n / 42 538, plan d'une jonque du Fu-Kien (cliché Musée national de la Marine)

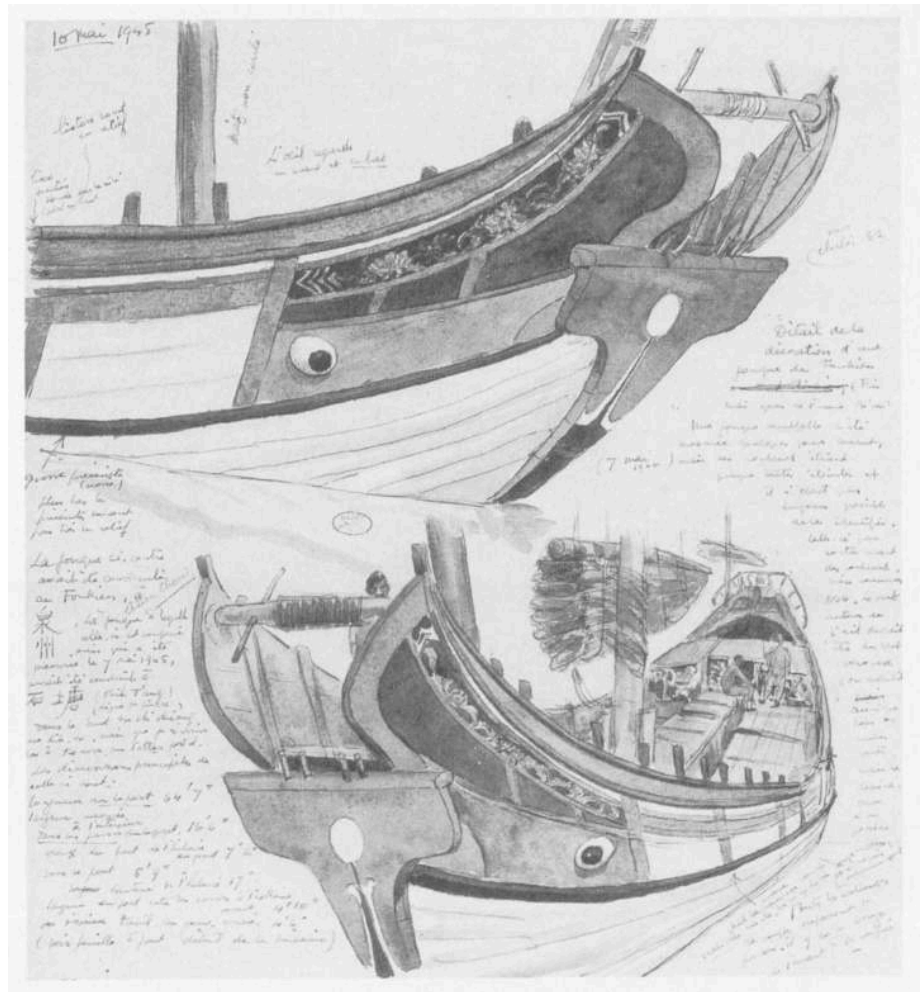

Figure 13. « cahier manuscrit » R9n / 42 538, feuille 2, p. 1 (cliché Musée national de la Marine) 


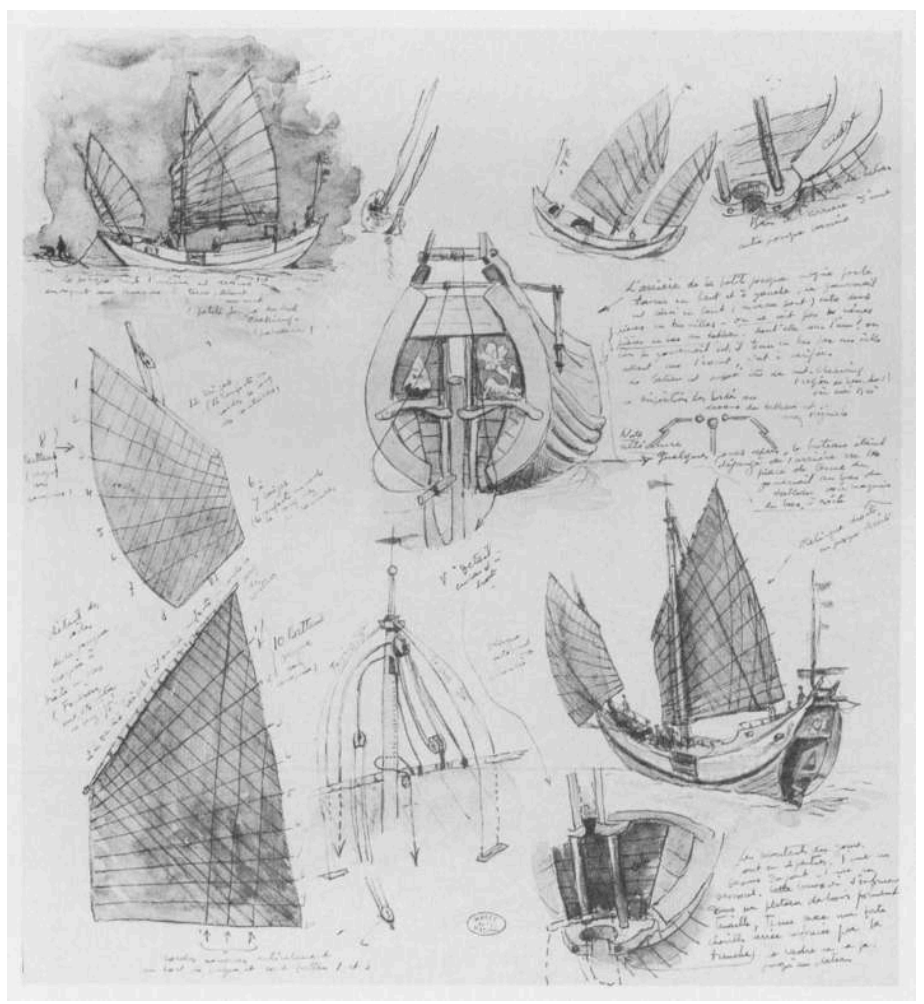

Figure 14. «cahier manuscrit » R9n / 42 538, feuille 2, p. 2 (cliché Musée national de la Marine)

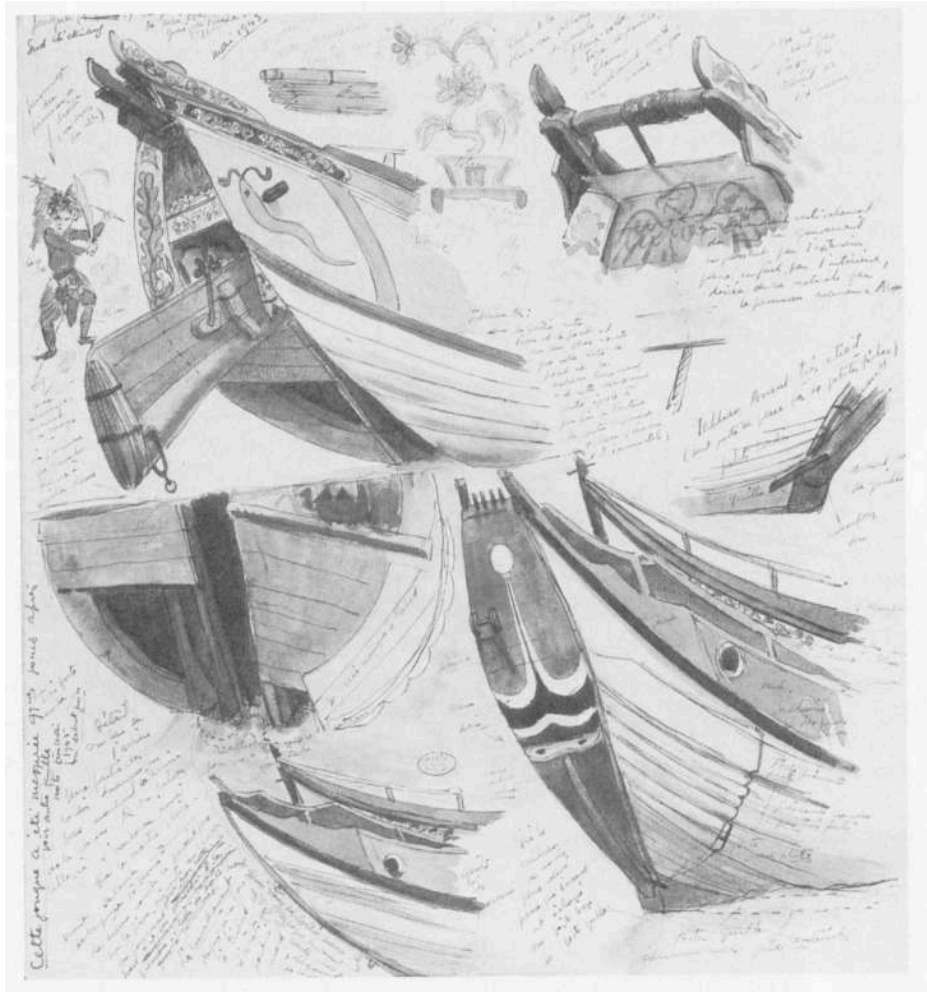

Figure 15. « cahier manuscrit » R9n / 42 538, feuille 2, p. 3 (cliché Musée national de la Marine) 


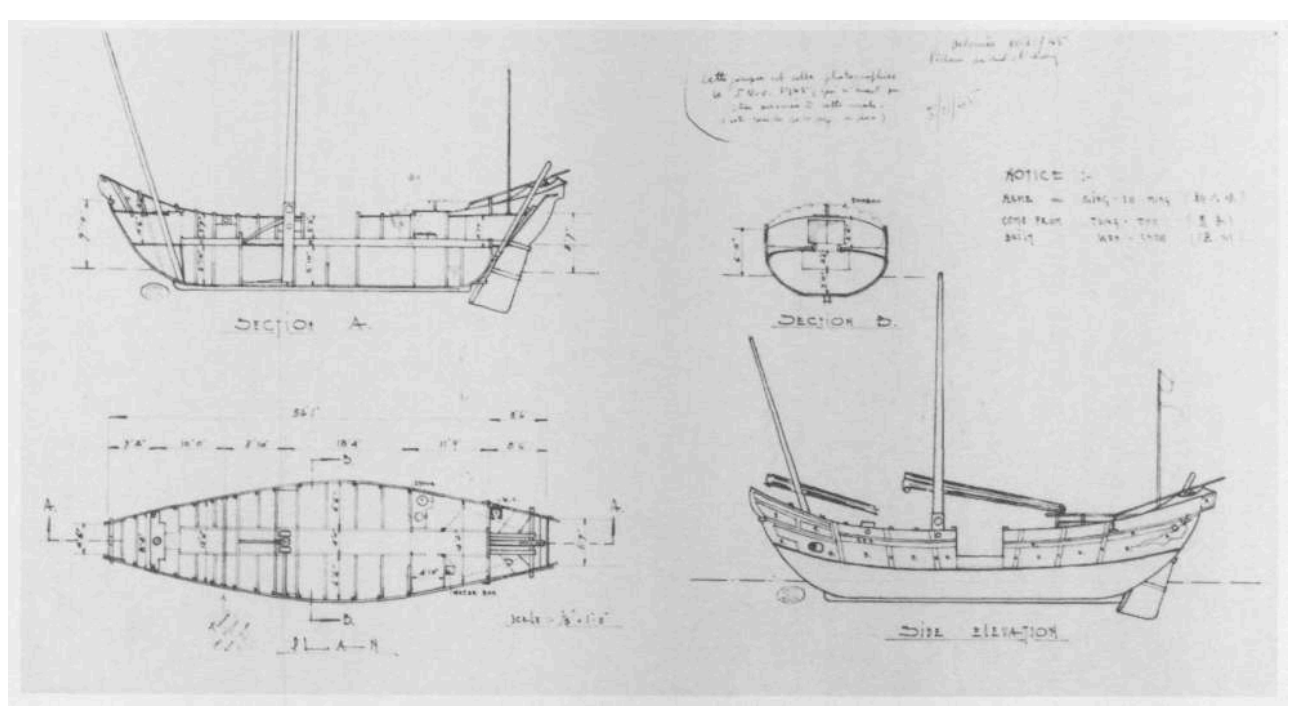

Figure 16. « cahier manuscrit » R9n / 42 538, plan d'une jonque du sud Ché-Kiang (cliché Musée national de la Marine)

Le troisième ensemble (une page) est un retour au thème majeur du « cahier » (fig. 17). Les notes et croquis de Sigaut portent, en effet, sur une " jonque du Foukien (env. 70' hors tout) amarrée quai de France et qui n'a pas été mesurée ». En quelques mots, il caractérise ce bâtiment : « Type classique, hiloire et serre bout à bout, roof bas en avant d'un château dans lequel se tient l'homme de barre sur le pont principal ». C'est sur l'exemple de cette jonque originaire de la province du Fu-Kien que s'achève ce « cahier» R9n / 42538 qui, tant au niveau de ses illustrations que de son texte, est représentatif, pour l'essentiel, des autres « cahiers manuscrits » (fig. 18).

Considérons maintenant, dans une troisième partie, de quelle manière la documentation recueillie par Sigaut s'insère dans un champ scientifique dont les bases ont été élaborées en France au milieu du XIX ${ }^{e}$ siècle. C'est en effet en 1843 que le capitaine de corvette -futur amiral- F. E. Pâris publie son Essai sur la construction navale des peuples extra-européens, une somme en deux tomes de grand format ${ }^{24}$, résultat de trois voyages de circumnavigation à bord de l'Astrolabe (1826-1829), la Favorite (1829-1832) et l'Artémise (1837-1840). Les « 800 plans ou dessins de pirogues ou navires de toutes les parties du monde" sont accompagnés de longs commentaires, indispensables et précieux compléments de l'atlas de planches. Cet ouvrage, le premier de ce genre édité dans le monde, repose sur la notion totalement nouvelle que les bateaux, et en particulier les bateaux traditionnels, sont susceptibles d'être des objets d'histoire, révélateurs de faits d'ordre culturel, au même titre que toute autre structure architecturale (palais, église, château...). Par ailleurs, il laisse entrevoir une méthode de travail qui peut se résumer en cinq termes: observer, dessiner, décrire, comparer, analyser (Rieth s/presse). Enfin, et il s'agit d'une autre dimension fondamentale de cette publication, y sont définis les fondements méthodologiques et scientifiques d'un territoire de recherche qui émergera, en tant que discipline des sciences de l'homme, au cours du XXe siècle : l'ethnographie nautique. C'est dans le prolongement de l'Essai et, d'une manière plus générale, d'une perception particulière des architectures navales traditionnelles, que se situent les « cahiers manuscrits » de Sigaut même si celui-ci ne mentionne pas explicitement dans ses «cahiers » cette parenté intellectuelle ${ }^{25}$. Le seul témoignage de cette relation consiste dans l'article que Sigaut a consacré à la vie et à l'oeuvre de Pâris. 


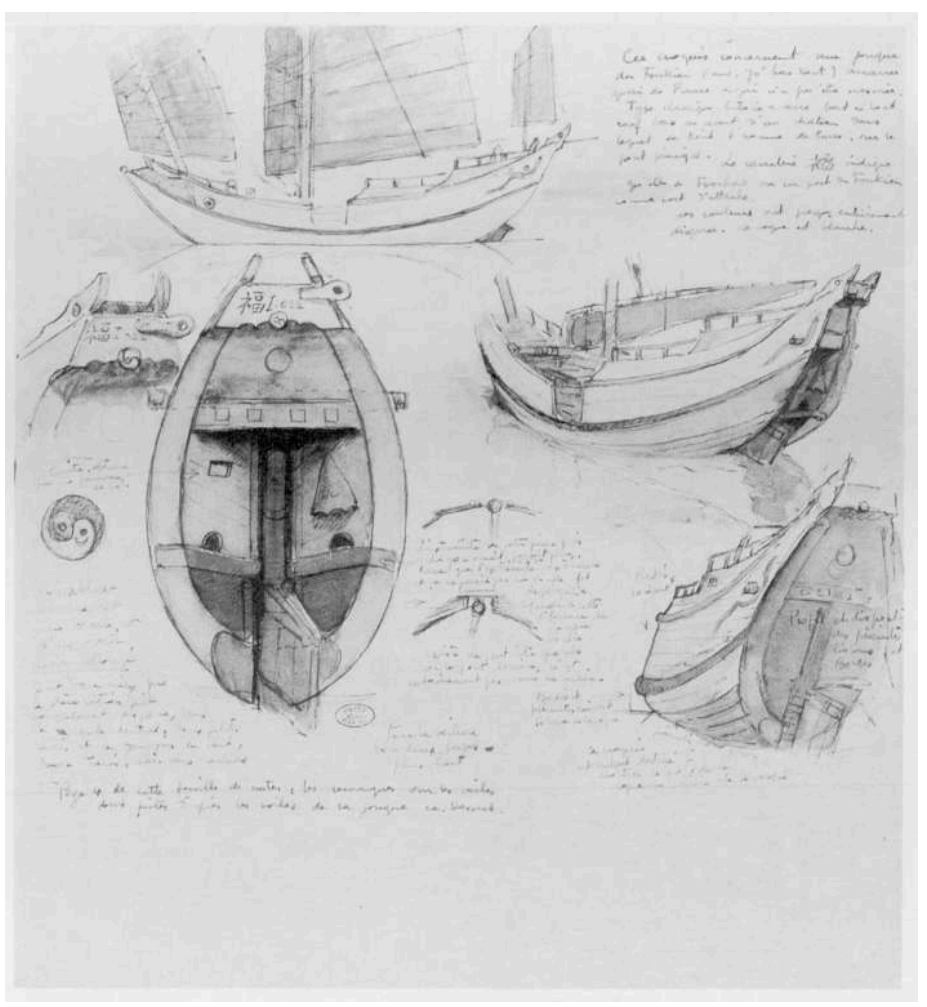

Figure 17. « cahier manuscrit » R9n / 42 538, feuille 2, p. 4 (cliché Musée national de la Marine)

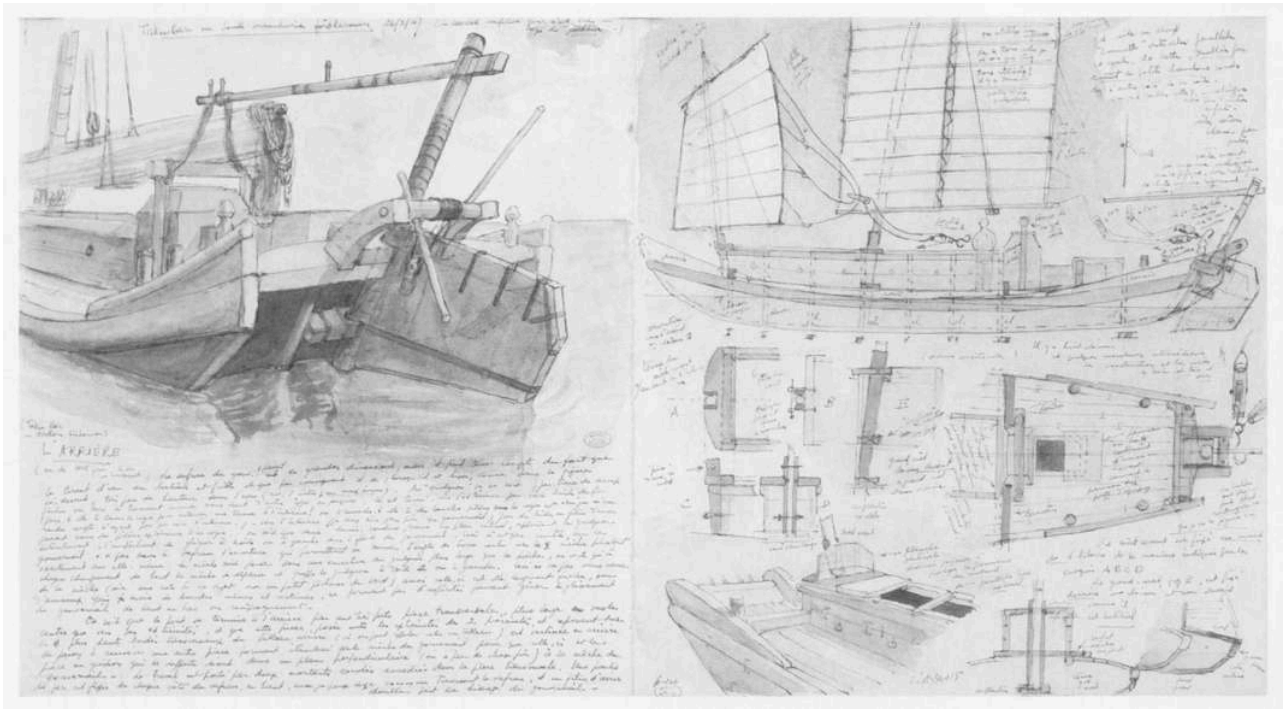

Figure 18. Jonque de pêche «Taku Bar ou South manchouria fischerman », 26 février 1947, «cahier manuscrit » R9n / 42510 (3) (cliché Musée national de la Marine)

Les deux hommes sont des chercheurs qui ont acquis par eux-mêmes une profonde culture historique, vaste et diversifiée pour Paris, spécialisée sur l'univers chinois pour Sigaut. D'une façon très différente, cette formation intellectuelle s'est développée dans le cadre d'une activité professionnelle en relation avec le milieu maritime. Chacun des deux hommes, l'un comme marin et l'autre comme agent commercial d'une grande compagnie de navigation, avait une connaissance et une expérience, d'un niveau certes différent, des navires. L'un et l'autre avaient également un intérêt -et une même passion- pour les bateaux exotiques que leur profession les avaient conduits à rencontrer. Sans doute, les motifs ayant poussé Pâris à s'engager dans ses recherches 
apparaissent-ils beaucoup plus affirmés et étendus, du point de vue de leur formulation tout au moins, que ceux ayant amené Sigaut à s'attacher à l'étude des jonques et des sampans de Chine. C'est ainsi que l'on ne discerne pas dans les "cahiers manuscrits » de Sigaut cette volonté, affichée à maintes reprises dans l'œuvre ethnographique de Pâris, de vouloir fixer la mémoire d'architectures navales traditionnelles et, à travers elles, de cultures nautiques (au sens large de l'expression) en voie de mutation pour la plupart, et de disparition pour certaines d'entre elles. En d'autres termes, la différence entre leurs approches pourrait se situer au niveau de l'étendue de leur projet. Paris avait, dès les années 1840-1845, une vision à long terme d'ordre scientifique et patrimonial qu'il amplifiera lors de sa nomination, en 1871, à la direction du musée naval du Louvre ${ }^{26}$. Sigaut, en revanche, semble avoir voulu témoigner, plus modestement, de la richesse d'un univers architectural qui s'offrait à son regard d'observateur privilégié de résidant en Chine. Toutefois, les maquettes qu'il fit construire et qu'il mit en dépôt au Museo storico navale de Venise ne sont-elles pas le révélateur d'une sensibilité patrimoniale proche de celle manifestée par Pâris? Les différences d'ambition scientifique entre les deux hommes ne seraient-elles pas, finalement, le reflet des différences existant entre la position d'un chercheur disposant d'un appui institutionnel (Pâris) et celle d'un "amateur» (Sigaut), travaillant sans moyens dans un pays en guerre?

39 Au-delà des dimensions de leur projet de recherche, Pâris et Sigaut se rejoignent sur bien des points. Ce sont d'abord des hommes de terrain, enquêtant selon les possibilités offertes par leurs activités professionnelles. Pour Pâris, ses lieux d'enquêtes se confondent avec ceux des escales des bâtiments à bord desquels il avait embarqué au cours de ses trois expéditions autour du monde. Pour Sigaut, son territoire d'étude est associé à celui de son travail à Shanghaï. Si le premier a eu la possibilité de recueillir une documentation ethnographique très vaste, à l'échelle des océans, mais au cours de séjours nécessairement réduits au temps des escales $^{27}$, le second a effectué ses observations sur une zone géographiquement et culturellement restreinte, mais, avantage certain, fréquentée quotidiennement pendant de nombreuses années.

Que ce soit sur le sable d'une plage du littoral du Bengale ou de l'archipel des Philippines pour Pâris ou le long du quai de France, à Shanghaï, pour Sigaut, leurs enquêtes reposaient sur deux actes de nature similaire : l'observation et le dessin. Pour autant, leur façon d'observer, guidés par une connaissance générale de l'architecturale navale, et de dessiner, facilitée par un talent artistique certain, demeurent personnelles. Paris apparaît ainsi beaucoup plus attaché que Sigaut à l'observation détaillée et technique du système architectural des bateaux, accordant une attention systématique à leurs caractéristiques structurelles, morphologiques, fonctionnelles. Il est vraisemblable que sa formation de marin d'une part, et son intérêt prononcé pour le domaine des techniques d'autre part ${ }^{28}$, ne sont pas étrangers à cette attitude. Sigaut, tout en n'excluant pas de ses observations les aspects techniques, tend cependant à s'attarder plus volontiers sur les caractéristiques morphologiques des coques, leurs décorations, ou encore les emménagements et les équipements du bord. Sa formation universitaire, juridique et commerciale, avait été, il est vrai, bien différente de celle d'un élève du Collège royal de la marine d'Angoulême.

41 Ces regards dissymétriques sur les bateaux se retrouvent dans la manière dont Pâris et Sigaut ont fixé graphiquement leurs observations. Les "cahiers manuscrits» sont, avant tout, de remarquables cahiers de dessins aquarellés, riches d'informations mais 
traduites d'une façon toujours esthétique. De ce point de vue, les illustrations des " cahiers manuscrits " rappellent, par leur forme ${ }^{29}$, les dessins aquarellés réalisés par Pâris lors de son embarquement à bord de la Favorite, entre 1829 et 1832, qui restituent les bateaux dans leur milieu d'utilisation (Rieth 1992). C'est, en l'occurrence, à partir de ces peintures que la plus grande partie des lithographies publiées dans l'Essai ont été réalisées.

À cette expression picturale, nécessairement subjective, mais non dénuée de rigueur du point de vue des données techniques, les deux hommes ont joint une documentation, a priori plus objective, sous la forme de relevés d'ensemble en plan, élévation et coupes, complétés dans les "cahiers" et dans l'Essai par de nombreux relevés de détails, particulièrement informatifs. Près d'un siècle séparant les deux hommes, Sigaut a largement utilisé la photographie en noir et blanc comme mode d'enregistrement complémentaire, se révélant, au demeurant, un photographe de grand talent. Cette collection de plusieurs milliers de clichés, conservée au Musée national de la Marine, représente une fabuleuse source, totalement inédite, dont l'étude reste à faire ${ }^{30}$.

À ce point de la réflexion, le fait important qui paraît devoir être souligné concerne l'association de deux types d'expression, l'un à connotation artistique (les dessins aquarellés) et l'autre à tendance technique (les relevés) qui, au regard des critères actuels des recherches ethnographiques, semblent devoir être opposés, le second excluant, au nom de la rigueur et du « nécessaire » recul objectif, le premier. Or, tant dans l'Essai ${ }^{11}$ que dans les "cahiers manuscrits", ces deux modes d'expression apparaissent, de notre point de vue, totalement complémentaires et contribuent, chacun à leur manière, à la construction d'un corpus documentaire doté d'un contenu scientifique affirmé. En d'autres termes, la sensibilité d'un croquis aquarelle n'efface nullement son apport technique qui, bien entendu, ne se confond pas avec celui d'un relevé architectural précisément coté.

Après l'observation et le dessin intervient, dans l'enquête, la description, troisième terme définissant la "méthode Pâris ». À cet égard, le texte des " cahiers manuscrits ", en dépit de son caractère provisoire sous la forme d'une suite de notes rédigées sommairement, semble présenter le même caractère de rigueur et de précision que les commentaires de l'Essai. Il est difficile, toutefois, de mesurer globalement cet aspect de l'œuvre de Sigaut dans la mesure ou seul un «cahier » a donné lieu à une rédaction définitive en vue de sa publication dans le Mariner's Mirror. Pour cette même raison, il est tout aussi malaisé d'évaluer le texte des " cahiers manuscrits » au regard des deux autres termes propres à la " méthode Paris » : comparer et analyser.

En référence à l'article sur un type de jonque de la Chine du nord, on constate qu'après une courte introduction situant le modèle de jonque étudié (construction dans les années 1895 à Chin-Kou, dans la province du Shan-Tung, structure présentant des indices d'une évolution architecturale à partir d'un radeau), Sigaut décrit en une série de courts paragraphes les principales caractéristiques du bâtiment: construction, écoutille principale, bordés, pont, poupe, cockpit, gouvernail, barre, cabine, ancres, bittes, mâts, voiles, embarcation. Dix dessins en noir et blanc (version dérivée des croquis aquarellés des "cahiers») et un plan accompagnent la description, claire et précise, de la jonque. Il s'agit, en fait, d'une très bonne monographie descriptive qui permet d'avoir une excellente connaissance de l'ensemble du bâtiment, même si les aspects structurels occupent, dans le cas présent, une place réduite (indication de la 
présence de douze cloisons, seuls renforts transversaux de la coque; absence de membrures et de baux).

Avec une écriture certes différente -le style des années quarante a évolué par rapport à celui du milieu du XIX ${ }^{e}$ siècle-, la manière de décrire cette jonque est proche de celle de Pâris. On retrouve le même soin apporté à définir, dans un vocabulaire spécialisé, sans pour autant être obscur, les caractéristiques du bâtiment. En revanche, les aspects comparatifs et analytiques demeurent presque totalement absents alors qu'ils sont très présents dans les textes de Pâris. Celui-ci prolonge fréquemment ses descriptions par des comparaisons entre des bateaux de même origine ou, au contraire, entre des unités de provenance différente dans le but de mettre en évidence des solutions architecturales -de structure ou de gréement- originales ou similaires. En outre, Pâris fait souvent référence au contexte historique et au cadre géographique au sein desquels s'insèrent les bateaux qu'il décrit dans la perspective de faire apparaître l'adaptation harmonieuse de l'architecture navale étudiée à un milieu naturel déterminé (plus ou moins contraignant), et à des besoins économiques particuliers. De la sorte, il dépasse les strictes limites de la monographie technique pour aboutir à une monographie aux dimensions ethnographiques. Chez Sigaut, les bateaux restent pour l'essentiel -mais c'est déjà un fait remarquable - des objets d'histoire des techniques. Chez Pâris, ils deviennent des objets d'histoire ${ }^{32}$. Il faut toutefois tempérer quelque peu ce jugement dans la mesure où les éléments d'appréciation correspondent dans un cas (l'Essai ) à une œuvre achevée et publiée et, dans un autre (les "cahiers»), à une documentation rédigée provisoirement et non mise en forme à l'exception d'un seul texte.

Au terme de cette évocation des « cahiers manuscrits » de Sigaut qui n'avait pour seule ambition, rappelons-le, que de présenter une source inédite, deux dernières remarques sont à faire. D'une part, cette documentation exceptionnelle par l'intérêt de son contenu et la qualité de son illustration mériterait, sans aucun doute, d'être analysée et publiée intégralement dans le cadre d'une édition commentée. D'autre part, la publication des "cahiers manuscrits ", sous la forme d'un livre d'érudition mais aussi d'un ouvrage d'art, serait, en vérité, un bel hommage rendu aux constructeurs et marins anonymes des jonques et des sampans dont Etienne Sigaut a fidèlement recueilli, au prix d'un immense travail, la mémoire entre les années 1943 et 1947, quai de France, à Shanghaï ou au cours d'un de ses voyages le long du littoral de la Chine.

\section{BIBLIOGRAPHIE}

Poujade, Jean, n.d., Traité d'ethnographie navale, ms dactylographié. Conflans-Sainte-Honorine : Musée de la Batellerie.

- 1948, Collection de documents d'ethnographie navale, d'archéologie navale, d'ethnographie terrestre, d'archéologie terrestre. Paris. Gauthier Villars

Rieth, Eric, 1992, Le Voyage de la Favorite. Textes et légendes. Arcueil : Anthèse.

- 1995, « Note sur un album du commandant Audemard », Neptunia 197, 1 : 17-20.

- 1993, Voiliers et pirogues du monde au début du XIX siècle. Paris : Du May. 
- s/presse « Observer, dessiner, décrire, comparer, analyser : une nouvelle méthode d'étude de l'architecture navale selon l'amiral Pâris (1806-1893) ", in Nouveaux regards sur l'histoire des techniques. Mélanges offerts à Marie-Claire Amouretti. Aix-en-Provence : MMSH/ Maisonneuve et Larose.

Sigaut, Etienne, 1960 « A northern type of Chinese junk », The Mariner's Mirror 46, 3 : 160-174. - 1961, « François-Edmond Paris french admiral », The Mariner's Mirror 47,4:255-270.

Waters, D.W., 1938 « Chinese Junks : The Autung Trader ", The Mariner's Mirror 24, 1 : 49-67.

- 1939, « Chinese Junks : The Pechili Trader », The Mariner's Mirror 25 : 62-87.

- 1940, «The Chinese Junks : an exception, the Tongkung », The Mariner's Mirror 26, 1 : 79-95.

- 1946, « The Chinese Junk : The Twaqo », The Mariner's Mirror 33, $1: 28-38$.

- 1955, « The Straight, and other, Chinese Yulohs », The Mariner's Mirror 41, $1: 60-61$.

- 1961, « The Chinese Junks : The Hangchow Bay Trader and Fisher », The Mariner's Mirror 47, 4 : 255-270.

Worcester, G.R.G., 1940, Junks and Sampans of the upper Yangtze. Shanghaï : The Maritime Customs/Ill, Miscellaneous Series n51., 1947-48, The Junks and Sampans of the Yangtze. A study in Chinese nautical research. Shanghaï : The Maritime Customs/Ill, Miscellaneous Series $n^{\circ}$ 53-54.

- 1948, « The Chinese War-Junk », The Mariner's Mirror 34, $1:$ 16-25.

- 1954, « The Amoy fishing boat », The Mariner's Mirror 40, $4: 304-308$.

- 1955a, « The Foot Boat of Shaohsing », The Manner's Mirror 41,3: 222-226.

- 1955b, « Paddle-wheel craft in China », The Mariner's Mirror 41, 4 : 340-341.

- 1956a, « The Origin and Observance of the Dragon Boat festival in China », The Mariner's Mirror

42, 2 : 127-131., 1956b, « Four small craft of Taiwan », The Mariner's Mirror 42, 4 : 308-310.

- 1958, "Some brief notes on fishing in China », The Mariner's Mirror 45, 2 :49-63.

- 1959, The Junkman Smiles. Londres : Chatto and Windus.

- 1961, « Six craft of Kwantung ", The Mariner's Mirror $2: 130-144$.

- 1961, « Fout junks of Kiangi », The Mariner's Mirror 47, 3 : 187-195.

\section{NOTES}

2. Etienne Sigaut est décédé à Forcalquier (Alpes-de-Haute-Provence), le 28 juillet 1983 ou 1988. Le doute est lié à la graphie ambiguë du dernier chiffre inscrit sur l'acte d'état civil.

3. La forme ancienne des noms des provinces chinoises utilisée par Sigaut dans ses «cahiers manuscrits » a été respectée chaque fois que sa documentation est citée.

4. «Attestation de E. Rey, chef du Service du Personnel Sédentaire et des Affaires Sociales de la Compagnie des Messageries Maritimes (27 septembre 1972)", réf.: R9c / 43146 (2), dossier personnel Sigaut, Musée national de la Marine, désormais cité M.N.M

5. M.N.M., réf. : R9c / 43148 et R9c / 43171.

6. Dans une lettre datée du 19 avril 1947 adressée à Sigaut par Worcester, celui-ci écrit : «I am very glad for your sake that you are out of China... ». Lettre conservée dans un cahier de notes de terrain de Sigaut, M.N.M., réf. : J 3515. Autre indice : les derniers « cahiers manuscrits » datent de 1947.

7. Les deux volumes ont donné lieu à une nouvelle édition, revue et augmentée sous le titre de : The Junks and Sampans of the Yangize, Annapolis, 1971.

8. Cf. notamment ses articles parus dans The Mariner's Mirror de 1948 à 1961, ainsi qu'un ouvrage publié à Londres en 1959.

9. E. Sigaut, « cahiers manuscrits », M.N.M., réf. : R9n /42 560.

10. E. Sigaut, « cahiers manuscrits », M.N.M., réf. : R9n / 42512 (4). 
11. E. Sigaut, « cahiers manuscrits », M.N.M., réf. : R9n /45 510 (6).

12. E. Sigaut, « cahiers manuscrits », M.N.M., réf. : R9n /42 533.

13. E. Sigaut, « cahiers manuscrits », M.N.M., réf. : R9n /42 517.

14. E. Sigaut, « cahiers manuscrits », M.N.M., réf. : R9n / 42561.

15. E. Sigaut, «cahiers manuscrits », M.N.M., réf. : R9n / 42510 (1). « Jonques, Chine du nord, 17.11.1944».

16. Il est curieux de constater que Sigaut, qui semble avoir consulté des documents originaux de Pâris, considère que le « $a$ » de son nom ne porte pas d'accent circonflexe alors que l'amiral signe, presque toujours, Paris avec un accent circonflexe sur le « $a$ »

17. Il s'agit des dimensions les plus fréquentes.

18. E. Sigaut, « cahiers manuscrits ", M.N.M., réf. : R9n / 42510 à R9n / 42546 ; R9n / 42553 à R9n /42 561; R9n /42 563. Le « cahier» R9n / 42510 comporte six numéros : 42510 (1) à (6). Chaque numéro correspond, en fait, à un "cahier». Cette même subdivision se retrouve dans le « cahier » R9n / 42512 (1) à (4). Compte tenu de la dispersion de la documentation de Sigaut dans plusieurs fonds du musée, le chiffre de cinquante-cinq «cahiers manuscrits » est à considérer avec une certaine réserve.

19. E. Sigaut, «cahiers manuscrits ", M.N.M., réf.: R9n / 42 519. Les dates de réalisation des croquis (1911-1929) correspondent aux deux séjours de Sigaut à Shanghaï comme employé de la Compagnie des Messageries Maritimes. Les années 1911-1912 sont celles de sa première affectation. La période 1923-1928 est celle de sa deuxième affectation à l'agence générale des Messageries.

20. Pour six « cahiers », une certaine imprécision demeure sur les dates de réalisation.

21. Neptunia, 1966, 84, $4: 40$.

22. Neptunia, 1966, 82, $2: 39$.

23. Trois pages dactylographiées témoignent de ce désir d'organiser les notes manuscrites. Sigaut écrit ainsi : "début de dactylographie des notes manuscrites dans le but de reclasser dans un ordre plus logique... Travail hélas juste commencé ». Réf. : B9C / 43149 (14), M.N.M.

24. Réédition des planches avec une analyse dans E. Rieth (1993).

25. Ce n'est pas le cas de Jean Poujade (1948) qui consacre un chapitre (p. 15-20) à «l'historique de l'oeuvre de l'amiral Pâris intitulée Souvenirs de marine conservés ".

26. La collection de maquettes réalisées sous sa direction, à partir de ses relevés, complétés par ceux de son fils Armand et de ses correspondants, représente l'expression muséographique de cette perspective à la fois scientifique et patrimoniale.

27. Avec les risques, inévitables, d'une approche parfois superficielle et incomplète.

28. Cet intérêt pour le domaine des techniques se manifeste, notamment, dans ses nombreuses publications consacrées à la propulsion à vapeur et à la construction métallique.

29. Au niveau des sujets traités, les croquis aquarellés de Sigaut sont différents de ceux de Pâris. Ce dernier représente, en règle générale, l'ensemble du bateau alors que Sigaut multiplie, parfois sous différents angles, les vues de détail.

30. Jérôme Legrand, documentaliste au Musée national de la Marine, chargé du fonds photographique, a entrepris un inventaire et une première analyse de cette collection.

31. Il s'agit des lithographies.

32. En dépit de ses immenses connaissances, Pâris se laisse parfois entraîner à des conclusions historiques discutables. 


\section{RÉSUMÉS}

Etienne Sigaut, agent de la compagnie des Messageries Maritimes à Shanghaï a réalisé entre 1943 et 1947 des cahiers de croquis inédits. Ces cahiers, conservés au Musée national de la Marine, sont consacrés aux différents modèles de jonques de commerce et de pêche observées dans le port de Shanghaï. Auteur d'un article sur l'œuvre de l'Amiral Pâris, Sigaut s'inscrit dans la continuité de celui-ci. Fondés sur des enquêtes personnelles et accordant une part importante à l'expression graphique, les cahiers de croquis constituent une remarquable série de monographies complétant les recherches menées par Audemard, Donnelly et Worcester, avec qui Sigaut a entretenu des relations étroites. Cette documentation est présentée en soulignant les méthodes de travail de Sigaut et leur apport à la connaissance de l'architecture navale chinoise

The object of this article is to present, as a preliminary approach, an unpublished work conserved by the National Maritime Museum of Paris. The notebooks of Etienne Sigaut (1887-1983) are an exceptional documentation of traditional chinese naval architecture. Before examining this documentation, we feel it to be important at first, to briefly situate the author of this work.

Etienne Sigaut, agente de la compañía de las Messageries Maritimes en Shangai entre 1943 y 1947, realizó varios cuardenos de croquis inéditos. Los cuardenos conservados en el Museo de la Marina están consagrados a los diferentes modelos de juncos de comercio y de pesca observados en el puerto de Shangai. Autor de un artículo sobre la obra del almirante Pâris, Sigaut se inscribe en su continuidad. Fundados sobre encuestas personales y acordando la parte importante a la expresión gráfica, los cuadernos de croquis constituyen una notable serie de monografías que completan las investigaciones llevadas a cabo por Audemard Donnelly - y Worcester, con quien Sigaut ha mantenido estrechas relaciones. Esta documentación está presentada subrayando dos métodos de trabajo de Sigaut y su aporte al conocimiento de la arquitectura naval china.

\section{INDEX}

Mots-clés : architecture navale, Chine, jonques, sampans, Sigaut (E.)

\section{AUTEUR}

\section{ERIC RIETH}

CNRS-LAMOP, Musée national de la Marine, Palais de Chaillot, 75116 Paris 